\title{
QUBIC: Observing the Polarized Microwave Sky over the Puna
}

\section{QUBIC Collaboration:}

Beatriz García1, Diego Harari², Claudia Scóccola³ ${ }^{3}$ Peter Ade ${ }^{4}$, José G. Alberro ${ }^{3}$, Alejandro Almela ${ }^{1}$, Giorgio Amico ${ }^{5}$, Horacio Arnaldi², Didier Auguste ${ }^{6}$, Jonathan Aumont ${ }^{7}$, Susanna Azzoni $^{8}$, Stefano Banfi ${ }^{9,10}$, Paola Battaglia ${ }^{11}$, Elia Battistelli ${ }^{5,12}$, Alessandro Baú ${ }^{9,10}$, Benoit Bélier $^{13}$, David G. Bennett ${ }^{14}$, Laurent Bergé ${ }^{15}$, Jean-Philippe Bernard ${ }^{16}$, Marco Bersanelli ${ }^{17,18}$, Marie-Anne Bigot Sazy ${ }^{19}$, Nathan Bleurvacq ${ }^{19}$, Juan Bonaparte ${ }^{20}$, Julien Bonis $^{6}$, Asdrúbal Bottani ${ }^{3}$, Emory Bunn ${ }^{21}$, David Burke ${ }^{14}$, Daniele Buzi ${ }^{5}$, Alessandro Buzzelli ${ }^{22,23}$, Francesco Cavaliere ${ }^{17,18}$, Pierre Chanial ${ }^{19}$, Claude Chapron ${ }^{19}$, Romain Charlassier $^{19}$, Fabio Columbro ${ }^{5,12}$, Gabriele Coppi ${ }^{24}$, Alessandro Coppolecchia ${ }^{5,12}$, Giuseppe D’Alessandro 5,12 , Paolo de Bernardis ${ }^{5,12}$, Giancarlo De Gasperis ${ }^{22,23}$, Stéphane Dheilly $^{19}$, Michele De Leo ${ }^{5,25}$, Marco De Petris ${ }^{5,12}$, Andrés Di Donato ${ }^{20}$, Louis Dumoulin ${ }^{15}$, Alberto Etchegoyen ${ }^{1}$, Adrián Fasciszewski ${ }^{20}$, Luciano P. Ferreyro ${ }^{1}$, Diego Fracchia1 , Cristian Franceschet ${ }^{17,18}$, Martín Miguel Gamboa Lerena ${ }^{3}$, Kenneth Ganga ${ }^{19}$, Manuel E. García Redondo ${ }^{1}$, Michel Gaspard ${ }^{6}$, Amanda Gault ${ }^{26}$, Donnacha Gayer ${ }^{14}$, Massimo Gervasi ${ }^{9,10}$, Martin Giard $^{16}$, Valerio Gilles ${ }^{5}$, Yannick Giraud Héraud ${ }^{19}$, Mariano Gómez Berisso ${ }^{2}$, Manuel González $^{2}$, Marcin Gradziel ${ }^{14}$, Laurent Grandsire ${ }^{19}$, Jean-Christophe Hamilton ${ }^{19}$, Vic Haynes $^{8}$, Sophie Henrot-Versillé ${ }^{\text {, Duc Thuong Hoang }}{ }^{19,27}$, Federico Incardona ${ }^{17,18}$, Eric Jules $^{6}$, Jean Kaplan ${ }^{19}$, Andrei Korotkov ${ }^{28}$, Christian Kristukat ${ }^{20,29}$, Luca Lamagna ${ }^{5,12}$, Sotiris Loucatos $^{19}$, Thibaut Louis ${ }^{6}$, Raúl Horacio Luterstein ${ }^{20}$, Bruno Maffei ${ }^{7}$, Stefanos Marnieros ${ }^{15}$, Silvia Masi, ${ }^{5,12}$, Angelo Mattei ${ }^{12}$, Andrew May ${ }^{8}$, Mark McCulloch ${ }^{8}$, Maria Clementina Medina $^{30}$, Lorenzo Mele ${ }^{5}$, Simon J. Melhuish ${ }^{8}$, Aniello Mennella ${ }^{17,18}$, Ludovic Montier ${ }^{16}$, Louise Mousset ${ }^{19}$, Luis Mariano Mundo ${ }^{3}$, John Anthony Murphy ${ }^{14}$, James David Murphy ${ }^{14}$, Federico Nati ${ }^{9}{ }^{10}$, Créidhe O'Sullivan ${ }^{14}$, Emiliano Olivieri ${ }^{15}$, Alessandro Paiella ${ }^{5,12}$, François Pajot $^{16}$, Andrea Passerini ${ }^{9,10}$, Hernan Pastoriza ${ }^{2}$, Alessandro Pelosi ${ }^{12}$, Camille Perbost ${ }^{19}$, Maurizio Perciballi ${ }^{12}$, Federico Pezzotta ${ }^{17,18}$, Francesco Piacentini ${ }^{5,12}$, Michel Piat ${ }^{19}$, Lucio Piccirillo $^{8}$, Giampaolo Pisano ${ }^{4}$, Manuel Platino ${ }^{1}$, Gianluca Polenta ${ }^{31}$, Damien Prêle ${ }^{19}$, Roberto Puddu ${ }^{32}$, Damien Rambaud ${ }^{16}$, Pablo Ringegni ${ }^{3}$, Gustavo E. Romero ${ }^{30}$, Maria Salatino ${ }^{33}$, Juan M. Salum ${ }^{1}$, Alessandro Schillaci ${ }^{34}$, Stephen P. Scully ${ }^{14,35}$, Sebastiano Spinelli ${ }^{9}$, Guillaume Stankowiak ${ }^{19}$, Michail Stolpovskiy ${ }^{19}$, Federico Suarez ${ }^{1}$, Andrea Tartari ${ }^{36}$, Jean Pierre Thermeau ${ }^{19}$, Peter Timbie ${ }^{26}$, Maurizio Tomasi ${ }^{17,18}$, Steve A. Torchinsky ${ }^{19}$, Matthieu Tristram ${ }^{6}$, Carole E. Tucker ${ }^{4}$, Gregory S. Tucker ${ }^{28}$, Sylvain Vanneste ${ }^{6}$, Daniele Viganó $^{17}$, Nicola Vittorio ${ }^{22,23}$, Fabrice Voisin ${ }^{19}$, Robert Watson ${ }^{8}$, Francois Wicek ${ }^{6}$, Mario Zannoni ${ }^{9,10}$ and Antonio Zullo ${ }^{12}$

\footnotetext{
${ }^{1}$ Instituto de Tecnologías en Detección y Astropartículas (CNEA-CONICET-UNSAM), Buenos Aires B1650, Argentina

${ }^{2}$ Centro Atómico Bariloche and Instituto Balseiro, CNEA, San Carlos de Bariloche R8402AGP, Argentina

${ }^{3}$ Universidad Nacional de la Plata, La Plata B1900FWA, Argentina

${ }^{4}$ School of Physics and Astronomy, Cardiff University, Cardiff CF10 3AT, UK

${ }^{5}$ Department of Physics, Università di Roma La Sapienza, 00185 Roma, Italy

${ }^{6}$ Laboratoire de l'Accélérateur Linéaire (CNRS-IN2P3), 91898 Orsay, France

${ }^{7}$ Institut d'Astrophysique Spatiale (CNRS-INSU), 91405 Orsay

${ }^{8}$ School of Physics \& Astronomy, University of Manchester, Manchester M13 9PL, UK

${ }^{9}$ Department of Physics, Università di Milano Bicocca, 20126 Milano, Italy
} 
${ }^{10}$ Istituto Nazionale di Fisica Nucleare Milano Bicocca Section, 20126 Milano, Italy

${ }^{11}$ Istituto Nazionale di Astrofisica/OAS Bologna, 40129 Bologna, Italy

${ }^{12}$ Istituto Nazionale di Fisica Nucleare Roma 1 Section, 00185 Roma, Italy

${ }^{13}$ Centre de Nanosciences et de Nanotechnologies, 91120 Palaiseau, France

${ }^{14}$ Department of Experimental Physics, National University of Ireland, Mariavilla, Maynooth $99 \mathrm{MX}+\mathrm{QH}$, Ireland

${ }^{15}$ Centre de Spectrométrie Nucléaire et de Spectrométrie de Masse (CNRS-IN2P3), 91405 Orsay, France

${ }^{16}$ Institut de Recherche en Astrophysique et Planétologie (CNRS-INSU), 31028 Toulouse, France

${ }^{17}$ Dipartimento di Fisica, Università degli Studi di Milano, 20133 Milano, Italy

${ }^{18}$ Istituto Nazionale di Fisica Nucleare Milano 1 Section, 20133 Milano, Italy

${ }^{19}$ AstroParticule et Cosmologie (CNRS-IN2P3), 75013 Paris, France

${ }^{20}$ Comisión Nacional De Energia Atómica, C1429BNP Buenos Aires, Argentina

${ }^{21}$ Department of Physics, Richmond University, Richmond, VA 23173, USA

${ }^{22}$ Dipartimento di Fisica, Università di Roma Tor Vergata, 00133 Roma, Italy

${ }^{23}$ Istituto Nazionale di Fisica Nucleare Roma Tor Vergata section, 00133 Roma, Italy

24 Department of Physics and Astronomy, University of Pennsylvania, Philadelphia 19104, USA

${ }^{25}$ Department of Physics, University of Surrey, Guildford GU2 7XH, UK

${ }^{26}$ Department of Physics, University of Wisconsin, Madison, WI 53706, USA

27 University of Science and Technology of Hanoi (USTH), Vietnam Academy of Science and Technology (VAST), Hanoi 10000, Vietnam

${ }^{28}$ Department of Physics, Brown University, Providence, RI 02912, USA

${ }^{29}$ Escuela de Ciencia y Tecnología, Universidad Nacional de San Martín, San Martin 1650, Argentina

${ }^{30}$ Instituto Argentino de Radioastronomía, Berazategui 1880, Argentina

${ }^{31}$ Agenzia Spaziale Italiana, 00133 Rome, Italy

32 Instituto de Astrofísica and Centro de Astro-Ingeniería, Pontificia Universidad Católica de Chile, Santiago 782046, Chile

${ }^{33}$ Kavli Institute for Particle Astrophysics and Cosmology and Physics Department, Stanford University, Stanford, CA 94305 USA

${ }^{34}$ Department of Physics, California Institute of Technology, Pasadena, California 91125, USA

${ }^{35}$ Institute of Technology Carlow, Ireland

${ }^{36}$ Istituto Nazionale di Fisica Nucleare Pisa Section, 56127 Pisa, Italy

Correspondence: harari@cab.cnea.gov.ar

\begin{abstract}
QUBIC (Q\&U Bolometric Interferometer for Cosmology) is an experiment designed to measure the polarization of the cosmic microwave background (CMB), the relic radiation from the BigBang. Detailed measurements of tiny temperature anisotropies in the CMB shaped our understanding of the early Universe. Accurate measurements of its polarization may reveal even earlier features, in particular the presence of gravitational waves with primordial origin. Such measurements can probe inflationary cosmological models, which postulate that quantum effects during an accelerated expansion at the earliest stages after the Big-Bang produced gravitational waves along with the density fluctuations that later seeded galaxy formation. QUBIC will join other international efforts currently pursuing this goal using a novel approach, which combines the sensitivity of bolometric detectors with the control of systematic effects provided by interferometry. After its current calibration phase is completed a technological demonstrator already constructed in European laboratories will be deployed in Alto Chorrillos, a site in the Puna near San Antonio de los Cobres, Salta, Argentina. The complete first module of the instrument is planned to be installed during 2020. We review the scientific goals of the project, the instrument design and its expected performance.
\end{abstract}

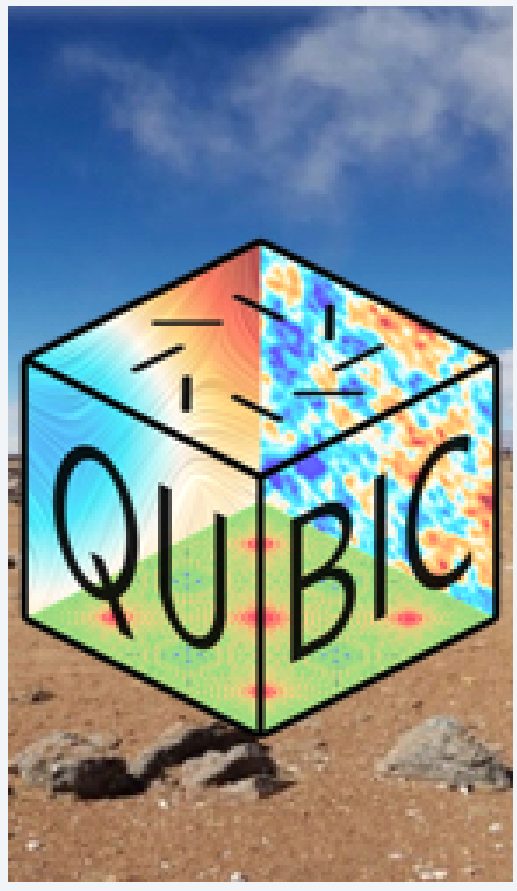

\title{
Keywords:
}

bolometric interferometry, cosmic microwave background, cosmology, gravitational waves, polarization, transition-edge sensors 


\section{Introduction}

The oldest light in the Universe is the Cosmic Microwave Background (CMB) radiation, discovered by Penzias and Wilson in 1965 [1]. It has a thermal distribution with a temperature of $2.725 \mathrm{~K}$. It brings us information about the conditions in the Universe almost 14 billion years ago, when no planets, stars or galaxies had yet formed. This was the time, 380,000 years after the Big-Bang, when the Universe had expanded enough so that the CMB temperature dropped down to the point that electrons and protons could combine into neutral atoms, from which photons scatter much less efficiently than from free electrons. The CMB photons bring us a picture of the Universe at such early times.

Tiny variations in the temperature of the CMB that arrives from different directions in the sky have been measured with increased precision since 1992 [2]. They are interpreted as the manifestation of small primordial inhomogeneities in the matter density that acted as seeds for the formation of galaxies and other large scale structures in the Universe. Their measurement allows a high-precision determination of the parameters of the current cosmological model [3].

The CMB was predicted to be linearly polarized [4]. The polarization was imprinted by anisotropic Thomson scattering during the last few interactions of $\mathrm{CMB}$ photons with free electrons, a process somewhat similar to how light is polarized after reflection off a surface. This small effect was experimentally verified for the first time in 2002 by the Degree Angular Scale Interferometer (DASI), located at the South Pole [5]. The process that imprinted the polarization was mostly driven by density inhomogeneities, responsible also for most of the CMB anisotropy. Gravitational waves can also leave their imprint in the $\mathrm{CMB}$ polarization $[6,7,8]$. A method was devised to disentangle what fraction of the polarization originates from gravitational waves $[9,10]$. It is based upon a decomposition of the polarization properties into $E$-modes, invariant under reflections, and $B$-modes, that change sign under a reflection. Scalar (density) fluctuations at first order generate $E$-modes only, while tensor (gravitational waves) fluctuations can produce both. The $E$-modes are much larger than the expected $B$-modes, and have been measured with increasing accuracy since 2002, providing further confirmation of the Big-Bang paradigm and increasing the precision on the determination of cosmological parameters. On the contrary, $B$-modes imprinted by gravitational waves have not been observed yet.

$B$-modes in the CMB polarization have been observed at relatively small angular scales, of the order of a few arc minutes $[11,12]$. These $B$-modes were predicted to exist as a consequence of weak gravitational lensing, the distortion of photon trajectories away from the straight line in an inhomogeneous gravitational field. Gravitational lensing rotates primordial $E$-modes into $B$-modes [13]. Measurement of these "secondary" $B$-modes is a great accomplishment, since an accurate mapping of the lensing effect provides insight into the distribution of dark matter on large scales, and may even allow an indirect measurement of the mass of the neutrinos.

Primordial $B$-modes, with an amplitude that is expected to peak at an angular scale of the order of one degree, are searched for with great expectation, since they would be indirect evidence for the existence of gravitational waves with cosmological wavelengths, that can not be detected directly with current instruments such as the Laser Interferometer Gravitational-Wave Observatory (LIGO) [14]. Primordial gravitational waves would be produced during an inflationary phase in the expansion of the early Universe. Inflation is currently the dominant paradigm to understand why the energy-density in the Universe is so close to the critical value corresponding to spatial-flatness, and why otherwise causally-disconnected regions when the CMB photons decoupled have almost the same temperature today [15, 16, 17]. It also provides a mechanism to generate tiny perturbations in the matter density that much latter seed galaxy formation. This same mechanism generates primordial gravitational waves, but inflationary models do not make a definite prediction for their amplitude, and consequently for the amplitude of the signal in $B$-modes. Observation of the footprint of gravitational waves in the $B$-modes of the $\mathrm{CMB}$ poses a great experimental challenge, partly due to their likely minuscule amplitude and also because their signature can be contaminated with polarized foregrounds of galactic origin.

Several experimental efforts are currently pursuing the search for primordial $B$-modes. Some are located at the South Pole $[18,19]$, that offers the driest site in the world and hence the best atmospheric conditions for the task. Others are in the Atacama desert in Chile [20, 21, 22, 23], the second driest site on Earth. We review in this article the QUBIC project $[24,25,26,27,28]$, that will soon join the search for primordial $B$-modes from the Puna in Salta, Argentina. QUBIC is a novel kind of instrument, that combines the sensitivity of bolometric detectors based on Transition-Edge Sensors [29] with the control of systematics granted by interferometric operation.

The outline of this article is as follows. In section 1 we review the scientific goals behind the quest for primordial $B$-modes. In section 2 we describe the design of the QUBIC instrument, with special emphasis on its most distinctive feature: its self-calibration properties based on interferometric operation. We review the characteristics of the site chosen for its installation in Alto Chorrillos, Salta. We summarize work on the detection and analysis chain and the expected performance of the instrument. We conclude in section 3 with a summary of the current status of the project, its schedule and future plans. 


\section{Footprints of primordial gravitational waves}

Gravitational waves were predicted to exist by Einstein in 1915, and a century elapsed until a first direct detection was possible, when LIGO measured the emission from the coalescence of binary black holes [14]. In the meantime, indirect evidence of their existence was available since 1974, when Hulse and Taylor verified that the rate of change in the period of a binary millisecond pulsar matched the expectation from the energy it should radiate away in gravitational waves[30]. The ongoing quest for primordial $B$-modes is analogous in the sense that while their measurement would not imply a direct detection, it would provide an unmistakable signature that gravitational waves with cosmological wavelengths existed well before any astrophysical object was formed.

\section{1. $E$ and B-modes}

Temperature anisotropies are usually characterized through an expansion over the celestial sphere in spherical harmonics

$$
T(\theta, \phi)=\sum_{\ell m} a_{\ell m}^{T} Y_{\ell m}(\theta, \phi)
$$

From measurements of temperature anisotropies we have gained a great knowledge about the Universe, such as its content in ordinary (baryonic) and dark matter, dark energy, its relatively negligible spatial curvature, the almost (but not quite) scale-invariant nature of the primordial matter inhomogeneities and their seemingly Gaussian distribution. We also learned that if primordial gravitational waves exist they contribute little to the observed anisotropies.

While this picture is quite compelling, there is still more to learn from measurements of the CMB polarization, particularly due to its sensitivity to the dynamics during the last few scatterings of the CMB photons. For instance, the observed anti-correlation of temperature and polarization confirms the coherence of fluctuations at scales larger than the horizon at the time when electrons and protons combined into neutral atoms [31]. This observation makes inflationary models of the early universe the most plausible explanation for the origin of density fluctuations, rather than for instance models based on topological defects, which do not produce fluctuations over scales larger than the horizon. Furthermore, the polarization properties of the $\mathrm{CMB}$ are particularly sensitive to the cosmological ionization and reionization history.
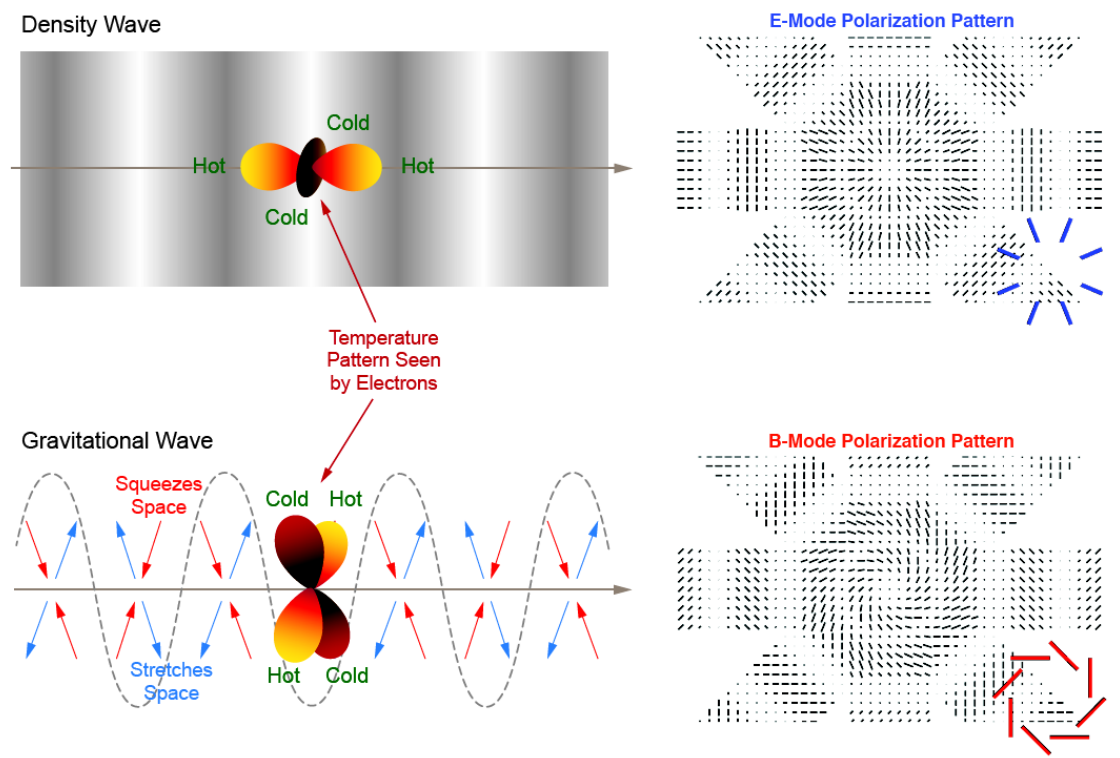

Figure 1. Illustration of polarization patterns induced by density waves compared to those by gravitational waves. Reproduced from [32].

Linear polarization is typically described in terms of Stokes parameters $Q$ and $U$, that can be expanded in spherical harmonics with spin 2 [33]:

$$
(Q \pm i U)=\sum_{\ell m} a_{\ell m}^{( \pm 2)} Y_{\ell m}^{( \pm 2)}
$$

Alternatively $E$ and $B$-modes are defined through the linear combinations $[9,10]$

$$
\begin{aligned}
& a_{\ell m}^{E}=-\left(a_{\ell m}^{(+2)}+a_{\ell m}^{(-2)}\right) / 2 \\
& a_{\ell m}^{B}=-\left(a_{\ell m}^{(+2)}-a_{\ell m}^{(-2)}\right) / 2 i .
\end{aligned}
$$


$E$-modes are parity invariant, while $B$-modes change sign under a parity transformation. Characterization of CMB polarization properties in terms of $E$ and $B$-modes is an invaluable source of information on different physical processes at work in the early Universe. $B$-modes can be used as a probe to infer if primordial gravitational waves drove partially the process that imprinted the polarization, in addition to density fluctuations. The origin of this possibility is illustrated in figure 1. Linear polarization can be imprinted in the CMB through Thomson scattering by electrons if the radiation has a quadrupolar anisotropy. The imprinted pattern of linear polarization differs depending on the type of motion of the electrons that scatter the radiation. Density waves produce a linear gradient in the gravitational potential that drives the motion of electrons, rotationally symmetric around the wave direction. Gravitational waves instead distort the gravitational potential differently in orthogonal directions perpendicular to their direction of propagation. The figure illustrates these different patterns in the rest frame of an electron. Shown also is the final polarization pattern after superposition of three crossing plane waves. Density waves do not imprint $B$-modes, while gravitational waves do.

Another contribution to $B$-modes comes from gravitational lensing that can convert a fraction of the CMB $E$-modes into $B$-modes[13]. The fluctuations in the gravitational potential of large scale structures distort the photon trajectories while preserving the orientation of the polarization, and thus alter the symmetry of the polarization pattern. The lensing contribution to $B$-modes can be disentangled and subtracted from a primordial signal through its different dependence on the angular scale as compared to that induced by gravitational waves, at least if the latter is not exceedingly smaller. It is also possible to generate $B$-modes by Faraday rotation of $E$-modes in primordial magnetic fields [34], but their frequency dependence would allow to clearly identify them if they actually existed. Density fluctuations can also produce $B$-modes through non-linear effects that generate secondary vector and tensor modes [35], but with a significantly reduced amplitude.

The major concern for contamination of a signal of primordial $B$-modes in addition to instrumental systematic effects are astrophysical foregrounds, particularly polarization by dust grains aligned by the galactic magnetic field and also synchrotron emission by relativistic electrons. They can in principle be accounted for through their angular power spectra and frequency dependence, distinct from that of the CMB. Measurements of CMB polarization at several frequencies are crucial for disentangling astrophysical foregrounds.

\subsection{Quantum effects during inflation}

An inflationary period in the early Universe $[15,16,17]$ may have been the source for the tiny perturbations in the matter density that much latter seeded galaxy formation. Inflation can both generate quantum fluctuations and also drive them to cosmological scales. All fields have quantum fluctuations in their vacuum state. Quantum fluctuations at small scales in the field $\phi$ that drives the inflationary expansion are stretched to cosmological wavelengths and transferred to the matter density as inhomogeneities at later stages, when the expansion is radiation or matter-dominated. The wavelength of the fluctuations is expanded by a factor larger than $10^{30}$ during inflation, which explains their cosmological size in spite of their quantum origin. Their amplitude is almost the same when their wavelength is comparable to the cosmological horizon. The fluctuations in the field $\phi$ driving inflation and those in gravitational waves $h$ have the same quantum origin and have comparable amplitude, determined by the value $H$ of the Hubble constant (the rate of expansion) during the inflationary phase:

$$
\left\langle h^{2}\right\rangle=\frac{G H^{2}}{2 \pi} \text { with } H^{2}=\frac{8 \pi G}{3} V(\phi) \Longrightarrow\left\langle h^{2}\right\rangle=\frac{V(\phi)}{M_{\text {Planck }}^{4}},
$$

where $G$ is Newton's constant, and $M_{\text {Planck }} \approx 10^{19} \mathrm{GeV}$ is Planck's mass. The relative intensity between the tensor and scalar fluctuations predicted by inflation is typically described in terms of the dimensionless quantity $r$ :

$$
r=\frac{\left\langle h^{2}\right\rangle}{\left\langle(\delta \rho / \rho)^{2}\right\rangle} .
$$

Here $\delta \rho / \rho$ are the relative density fluctuations, which are very well characterized by measurements of temperature anisotropies, and are of the order of $10^{-5}$. This is why a measurement of $r$ (the tensor-to-scalar ratio) is so relevant. It would reveal the presence of primordial gravitational waves, and determine the energy scale (the potential $V$ ) at which inflation took place:

$$
V^{1 / 4} \approx\left(\frac{r}{0.01}\right)^{1 / 4}\left(10^{16} \mathrm{GeV}\right) .
$$

A value of $r$ close to 0.01 would imply an energy scale close to that favoured theoretically for grand unified theories of fundamental interactions. Note, however, that there is no definite prediction for the value of $r$ in inflationary models.

The inhomogeneities in the matter distribution generated by the quantum fluctuations of the field $\phi$ depend also on the details of the potential $V(\phi)$ driving inflation

$$
\left\langle(\delta \rho / \rho)^{2}\right\rangle=\frac{G H^{2}}{32 \pi \varepsilon} \text { with } \varepsilon=-\frac{\dot{H}}{H^{2}} \approx \frac{M_{\text {Planck }}^{2}}{2}\left(\frac{V^{\prime}}{V}\right)^{2},
$$


where $\dot{H}$ is the time-derivative of the expansion rate, and $V^{\prime}$ is the derivative of the potential of the inflaton field, which determines the dependence of the density fluctuations with scale. Measurement of $r$ would then also provide additional insight into the shape of the potential driving inflation, not only its absolute scale [36].

\subsection{Bounds on primordial gravitational waves}

In 2014 the BICEP2 experiment, located just next to where DASI measured $E$-modes for the first time in 2002, made the first detection of $B$-modes in the sky at degree angular scales [37], compatible with a value $r=0.2$. It did so with an instrument operating at $150 \mathrm{GHz}$ and scanning a region of the sky in which the galactic contamination was expected to be negligible. Subsequent measurements and analysis, in particular correlating data from BICEP2 and Keck Array at $150 \mathrm{GHz}$ with that from the Planck satellite at $353 \mathrm{GHz}$, revealed [38] that the excess of $B$-modes observed at large angular scales was dominated by polarized emission by galactic dust. Figure 2 displays in the left panel the $95 \%$ confidence
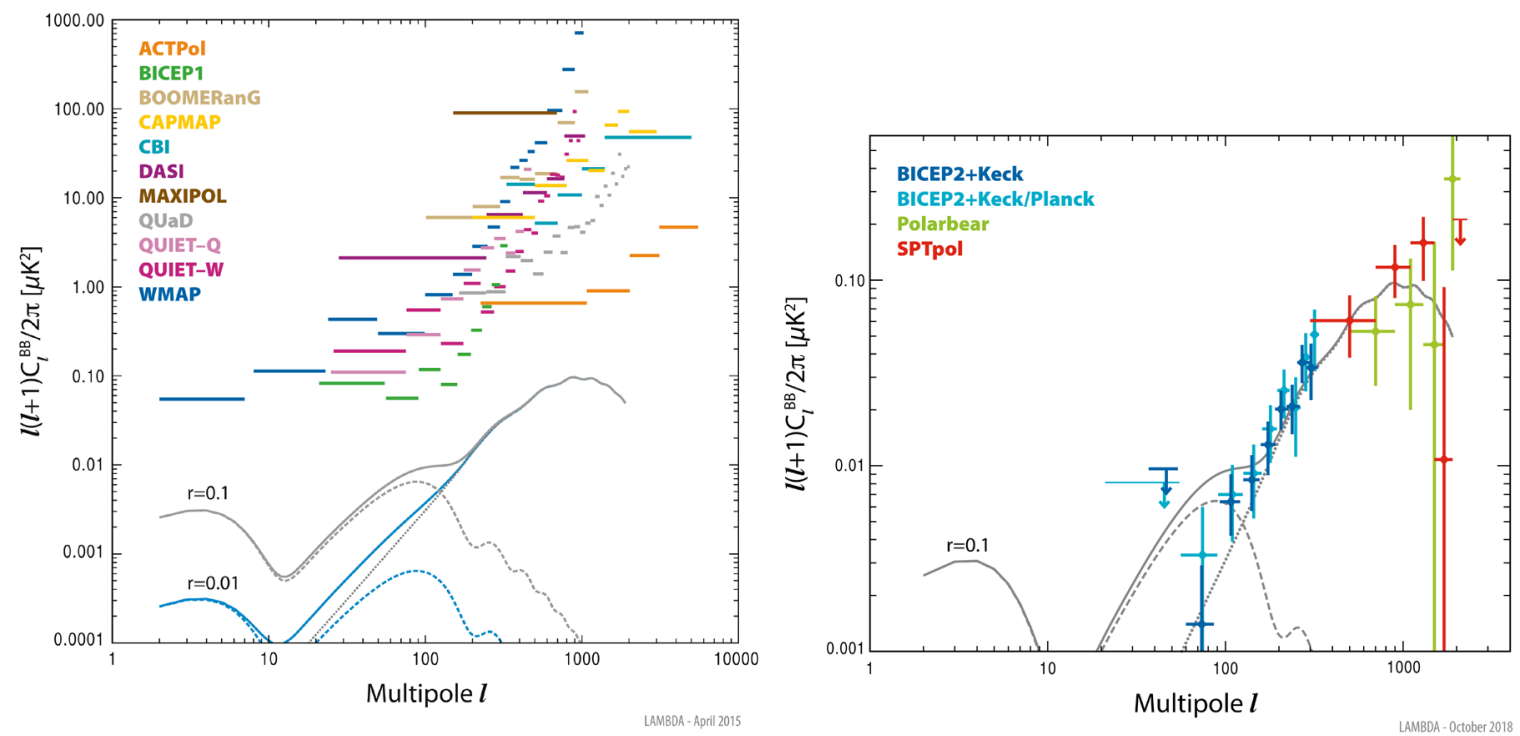

Figure 2. Left: $95 \%$ CL upper limits for the angular power spectrum in $B$-modes from different CMB experiments with no significant detections. Shown separately are the theoretical predictions for the contribution from lensing of $E$-modes (gray dotted line) and from primordial gravitational waves for $r=0.1$ (gray dashed) and $r=0.01$ (blue dashed lines). The solid lines correspond to the total power expected for $r=0.1$ (gray) and $r=0.01$ (blue). Right: observed $B$-modes from CMB experiments with significant detections. The lensing of $E$-modes into $B$-modes is detected with high significance, and at 95\% confidence level $r<0.06$. Figures reproduced from NASA-LAMBDA, https://lambda.gsfc.nasa.gov/.

level upper limits for $B$-mode power from different CMB experiments with no significant detections (as compiled in the NASA-LAMBDA site https://lambda.gsfc.nasa.gov/). Data from ACTPol, BICEP1, BOOMERanG, CAPMAP, CBI, DASI, MAXIPOL, QUaD, QUIET-Q, QUIET-W, and WMAP are included. Plotted is the rotationally invariant angular power spectrum $C_{\ell}^{B B}=\frac{1}{2 \ell+1} \sum_{m} a_{\ell m}^{B *} a_{\ell m}^{B}$ for the $B$-modes. The corresponding angular scale in terms of the multipole moment is about $1^{\circ}(180 / \ell)$. As reference theoretical predictions in a $\Lambda$ CDM cosmology with values of the tensor-to-scalar ratio $r=0.1$ (gray) and $r=0.01$ (blue) are shown. The dashed lines are the tensor contributions and the solid lines are the total power after addition of the expected signal from weak gravitational lensing of $E$-modes (gray dotted line). Note that the signal in $B$-modes from primordial gravitational waves peaks at degree angular scales $(\ell \approx 80)$ while that of lensing of $E$-modes peaks at significantly smaller angular scales $(\ell \approx 1000)$. A reionization bump is also predicted for the tensor modes at larger angular scales $(\ell<10)$. The right panel of figure 2 displays the $B$-mode power measured by experiments with significant detections. Results from BICEP2, Planck, POLARBEAR, and SPTpol are included. The BICEP2 + Keck data points show the CMB contribution after separation into CMB, dust, and synchrotron components. The BICEP2 + Keck/Planck data points show results with dust foreground subtraction based on measured cross-power between Planck and BICEP2 + Keck. The other points show results without any foreground subtraction.

The signal expected from rotation of $E$-modes into $B$-modes by weak gravitational lensing is detected with high significance, and at 95\% confidence level the tensor-to-scalar ratio is currently bound to be $r<0.06$ [39], which already disfavours the simplest inflationary models of the early Universe.

The quest for detection of primordial $B$-modes in the CMB continues to be a major experimental challenge. Accurate foreground contamination and removal of lensing effects for values as low as $r=0.001$ are challenging, but potentially feasible [40].

In what follows we describe the approach taken by QUBIC to confront the challenge of measuring primordial $B$-modes. 


\section{Measuring primordial B-modes with QUBIC}

\subsection{The QUBIC instrument}

QUBIC is an additive interferometer formed by different components, as schematized in figure 3. The detection system is

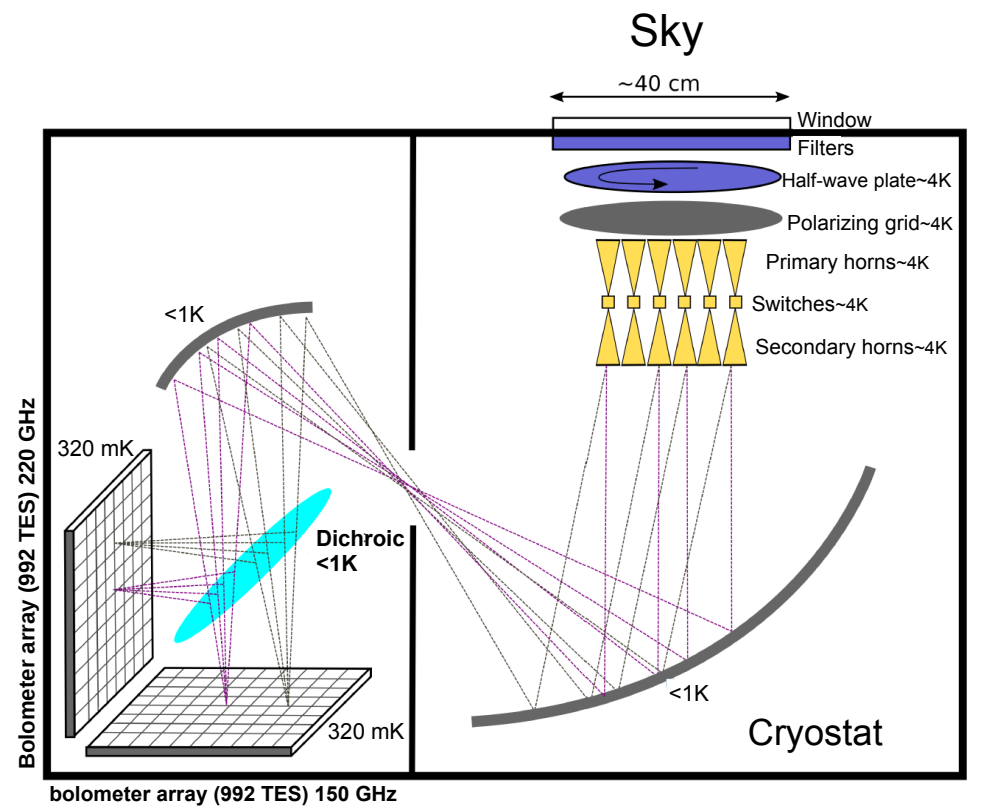

Figure 3. Schematics of the QUBIC instrument. Figure reproduced from [24].

contained inside a cryostat that is cooled down to $4 \mathrm{~K}$ using pulse-tubes. The microwave signals to be measured enter the cryostat through a $45 \mathrm{~cm}$ diameter window made of ultra-high molecular weight polyethylene [41] that provides excellent transmission and mechanical stiffness. After the window, filters ensure a low thermal load inside the cryostat. Following this are a rotating Half-Wave-Plate (HWP) that modulates the polarization, and then a polarizing grid that selects one of the two linear polarization components.

Right below the polarizing grid an array of 400 corrugated horns receives the incoming radiation and defines the baselines for the interferometric operation of the instrument. These primary horns are followed by secondary back-horns that re-emit the signal towards an optical combiner constituted of a dual-mirror system. This combiner allows the radiation coming from different directions to sum coherently in the various locations of the detector focal planes, thus producing the interference fringes pattern. A key part of QUBIC is an array of switches placed between the two feed-horn arrays, which can be used to exclude particular baselines when the instrument operates in self-calibrating mode, as described in section 2.4.1.

Between the mirrors and the focal planes a dichroic filter splits the signal into its $150 \mathrm{GHz}$ and $220 \mathrm{GHz}$ sub-bands, imaged onto orthogonal focal planes, each equipped with 1024 Transition-Edge-Sensors (TES) cooled down to $320 \mathrm{mK}$. Of the set of 1024 TES, 992 are exposed to the sky radiation, while the rest are used to characterize systematic effects. The sensors are read using a cryogenic readout system based on superconducting quantum interference devices (SQUIDs) and Si-Ge application-specific integrated circuits (ASIC) operating at $4 \mathrm{~K}$ with an unprecedented multiplexing factor equal to 128 [42, 43].

The TES technology is well developed and extensively used in several millimetric and sub-millimetric astronomical experiments. They have been chosen as detectors for the QUBIC first module. Other types of detectors such as Kinetic Inductance Detectors (KIDs) [44] will be considered for future QUBIC modules as they may offer an easier fabrication and readout, and larger scalability.

As mentioned before, the whole instrument will be integrated in a cryostat that needs to be operated without the use of cryogenic liquids in order to be usable in any remote observation site. The $4 \mathrm{~K}$ stage is therefore ensured thanks to a pulse-tube refrigerator, which must remain with unchanged cooling efficiency when the instrument is tilted in elevation in the range between $\pm 30^{\circ}$ and $70^{\circ}$ required to scan the region of the sky chosen for observation. The secondary and primary mirrors, dichroic filter and focal planes will be cooled down to $1 \mathrm{~K}$ with a dedicated ${ }^{4} \mathrm{He}$ adsorption fridge and integrated in a special mechanical enclosure, the " $1 \mathrm{~K}$ box". The purpose of the $1 \mathrm{~K}$ box in addition to thermal shielding is to assure the mechanical holding of these different parts. It was built stiff enough to guarantee the alignment of the optical components while the instrument scans the sky. The cryogenic stage for the detectors at $320 \mathrm{mK}$ will be ensured through a ${ }^{3} \mathrm{He} /{ }^{4} \mathrm{He}$ absorption fridge. The outer dimensions of the cryostat $(1.4 \mathrm{~m}$ in diameter and $1.6 \mathrm{~m}$ in height) allow for sufficient thermal insulation between the cryogenic instrument and the room-temperature shell. The total weight of the instrument is $800 \mathrm{~kg}$, 
and the weight of the overall internal structure that holds the horn array, the mirrors, the dichroic filter and the detectors has been limited to less than $150 \mathrm{~kg}$ in order to prevent too long a cooling time down to $1 \mathrm{~K}$.
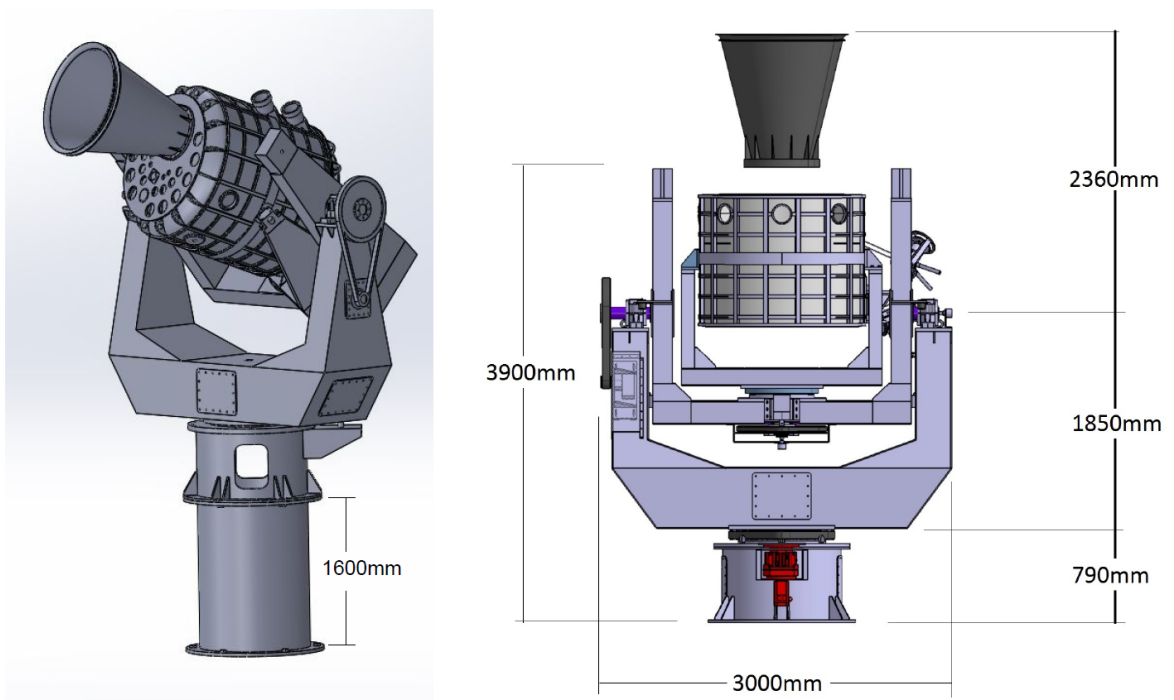

Figure 4. QUBIC assembly: cryostat with forebaffle on its mount (left); detail and dimensions (right).

QUBIC will host two arrays of bolometric detectors, operating respectively at $150 \mathrm{GHz}$ and $220 \mathrm{GHz}$, that will observe interference fringes formed by the back-to-back horns on the focal planes of the optical combiner. The image formed is the result of the interference from the sum of the fields emitted by each of the 400 apertures.

Interferometry offers an improved control of instrumental systematics with respect to direct imaging, because interference fringes from different baselines can be calibrated individually. This feature is made possible in QUBIC thanks to the electromagnetic shutters inserted between the primary and secondary horns. Each shutter consists of a blade operated by a solenoid magnet that can slide into a smooth circular waveguide, and acts as a RF switch.

In the self-calibration mode, pairs of horns are successively shut when observing an artificial partially polarized source. QUBIC will use for external self-calibration a source able to radiate a typical power of few $\mathrm{mW}$ through a feedhorn with a well-known beam, and a low level of cross-polarisation, typically less than $-30 \mathrm{~dB}$. The source is a microwave synthesizer operated around $10 \mathrm{GHz}$ followed by a multiplication chain which brings the frequency in the millimeter wave range to generate quasi-monochromatic signals in both QUBIC frequency bands. This external calibration source will be located in the far-field of the interferometer, at about $40 \mathrm{~m}$ from the instrument and it will be mounted on top of a tower near the instrument shelter inside an insulation box suitable to maintain the device within a specified temperature range. In addition, two carbon fiber sources located inside the cryostat, just next to to the array of back-to-back horns and switches, will also be used for calibration purposes [45].

The instrument will be installed on a mount, following a design as in Figure 4. It will be able to rotate $360^{\circ}$ in azimuth, from $30^{\circ}$ to $70^{\circ}$ in elevation, and $\pm 15^{\circ}$ around the optical axis, with a pointing accuracy better than 20 arcsec. A forebaffle with $1 \mathrm{~m}$ length and $14^{\circ}$ aperture will be used to reduce radiation from unwanted sources by more than $20 \mathrm{~dB}$ in directions between $20^{\circ}$ and $40^{\circ}$ away from the optical axis, and more than $40 \mathrm{~dB}$ beyond. A ground shield will also be included to minimize the brightness contrast between the sky and the ground.
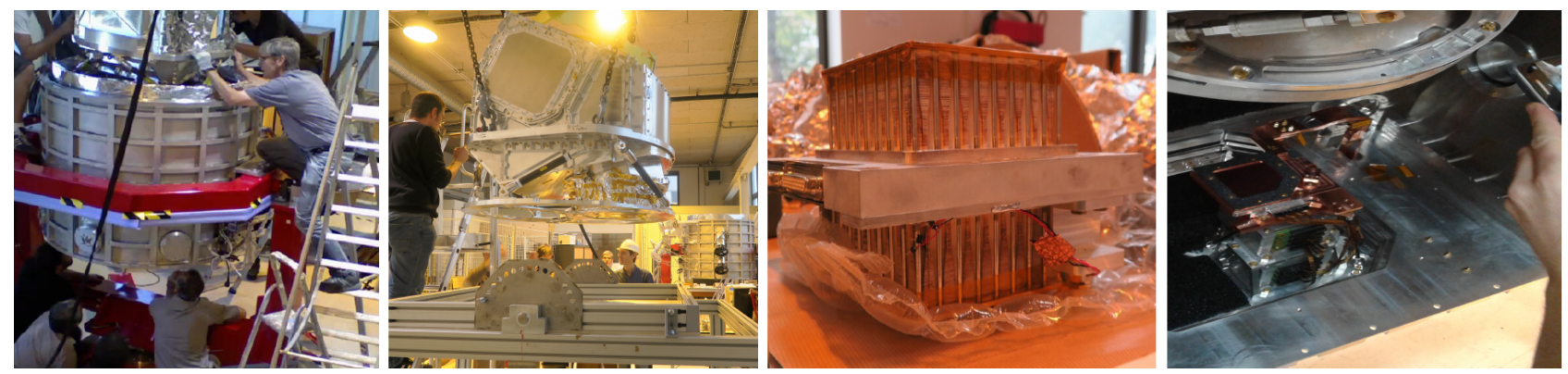

Figure 5. Pictures of various QUBIC components. From left to right: integration of the instrument inside the cryostat, $1 \mathrm{~K}$ box, array of 64 back-to-back horns with individual switches, and focal plane on top of the cryogenic detection chain.

The QUBIC instrument is currently in a phase of laboratory calibration of its so-called "Technological Demonstrator" (TD). The TD uses the cryostat and $1 \mathrm{~K}$ box built for the first module, but has a reduced focal plane and horn array and a smaller optical combiner compared to the full instrument. The TD has one-quarter of the TES focal plane (i.e. 256 pixels) 
operating at $150 \mathrm{GHz}$ and 64 back-to-back horns with their respective switches. The TD has already been integrated at the APC laboratories in Paris (see Figure 5) and is in the process of calibration, with very satisfactory preliminary results, as will be described in section 3. After calibration the TD will be sent to Argentina for installation on site while the construction of the complete first module continues. The TD was not designed to produce scientific results but will serve to demonstrate the feasibility of bolometric interferometry both in the laboratory as well as in the field.

A more detailed description of the QUBIC instrument can be found in its Technical Design Report in [27]. The QUBIC instrument is being built by an international collaboration with participating laboratories in France, Italy, UK, Ireland, USA and Argentina.

\subsection{The Alto Chorrillos site}

\subsubsection{The site}

The QUBIC instrument will be deployed in Alto Chorrillos, Salta, Argentina (24 $\left.11^{\prime} 11.7^{\prime \prime} \mathrm{S}, 66^{\circ} 28^{\prime} 40.8^{\prime \prime} \mathrm{W}\right)$ at 4,870 meters above the sea level, in what is known as the Puna de Atacama region. This location is $180 \mathrm{~km}$ away from the Chajnantor site in Chile where other millimeter-wave experiments and observatories are located (ALMA, APEX, Advanced ACTPol, POLARBEAR and CLASS) and offers similar atmospheric properties [46, 27]. It is next to the location of the Large Latin American Millimeter Array (LLAMA), a bi-lateral cooperation project between Argentina and Brasil that is installing a $12 \mathrm{~m}$ radiotelescope operating at millimeter and submillimeter wavelenghts (for more details see the article by G. Romero in this same volume). This location has been characterized during several years within the framework of the site selection process for the Cherenkov Telescope Array project, and for the LLAMA project, which has atmospheric requirements similar to those of QUBIC. We here summarize these and further studies that have been performed on the site properties, and the status of the infrastructure being developed.

The city nearest to the site is San Antonio de los Cobres, at an altitude of about 3,700 meters above sea level and 200 $\mathrm{km}$ from Salta city, the province capital. Originally a mining town, it now has about 7000 inhabitants, and in the last decade the town has witnessed the efforts of several international collaborations that have characterized the atmosphere of the area, searching for sites suitable for the installation of astrophysical experiments. The national route 40 crosses the region in the S-N direction. The most important nearby city is Salta, but it is also possible to access the area from San Salvador de Jujuy. The valley of San Antonio de los Cobres, which gives its name to the river that runs through it, unfolds parallel to the Andes. This valley culminates to the north in the depression of the Salinas Grandes, $80 \mathrm{~km}$ away from San Antonio. The site where QUBIC will be installed is named "Alto Chorrillos". It is located about $16 \mathrm{~km}$ in a straight line from the city

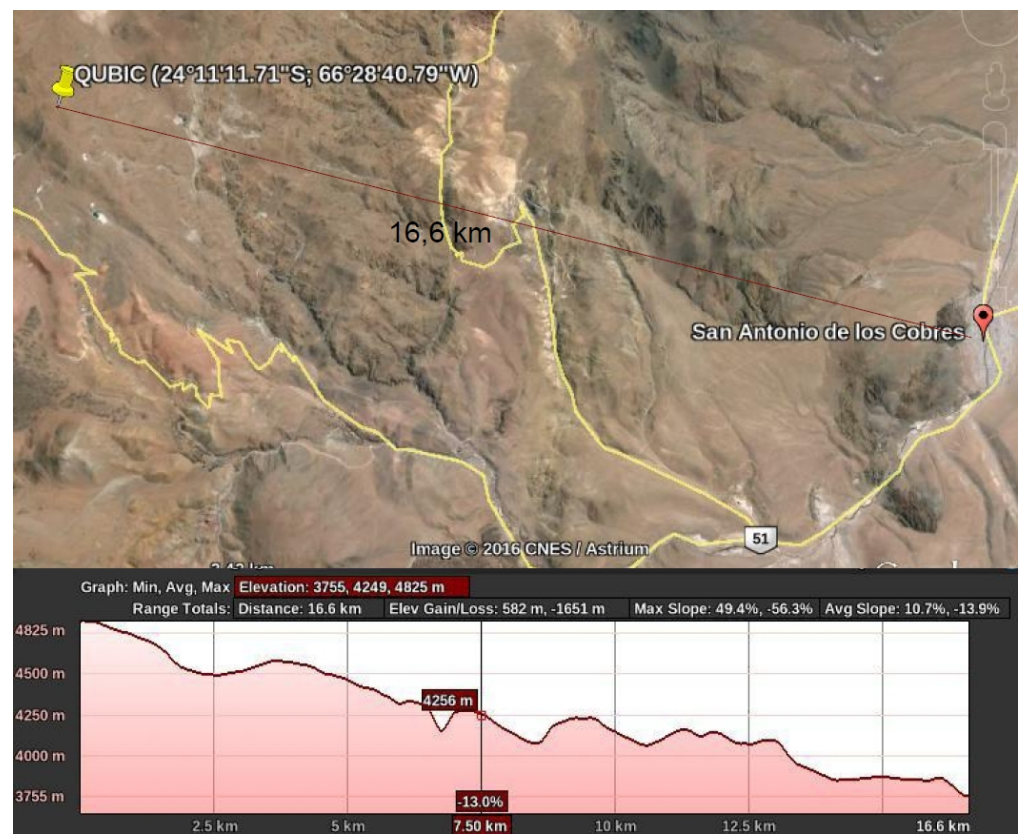

Figure 6. Qubic Site (top) and elevation profile (bottom). The positions of QUBIC and San Antonio de los Cobres are detailed as well as the distance between the sites. The elevation profile is such that it is possible to establish a direct link for communications.

of San Antonio de los Cobres, at 4870 meters above the sea level (see Figure 6) and in the same area where the LLAMA antenna will be installed. Alto Chorrillos is located within a reserve of 400 hectares allocated by the Government of the Province of Salta to Argentina's National Research Council (CONICET) for the installation of LLAMA. A flat area of about $125 \mathrm{~m}$ x $150 \mathrm{~m}$ will be used for the installation of QUBIC. A detailed map of this area is shown in the left panel of 
figure 7. It displays the site chosen for the installation of the first module, as well as those reserved for future modules. The right panel in the figure displays a simulation of an aerial view of the site including the shelter with the first module and the tower for the calibration source.
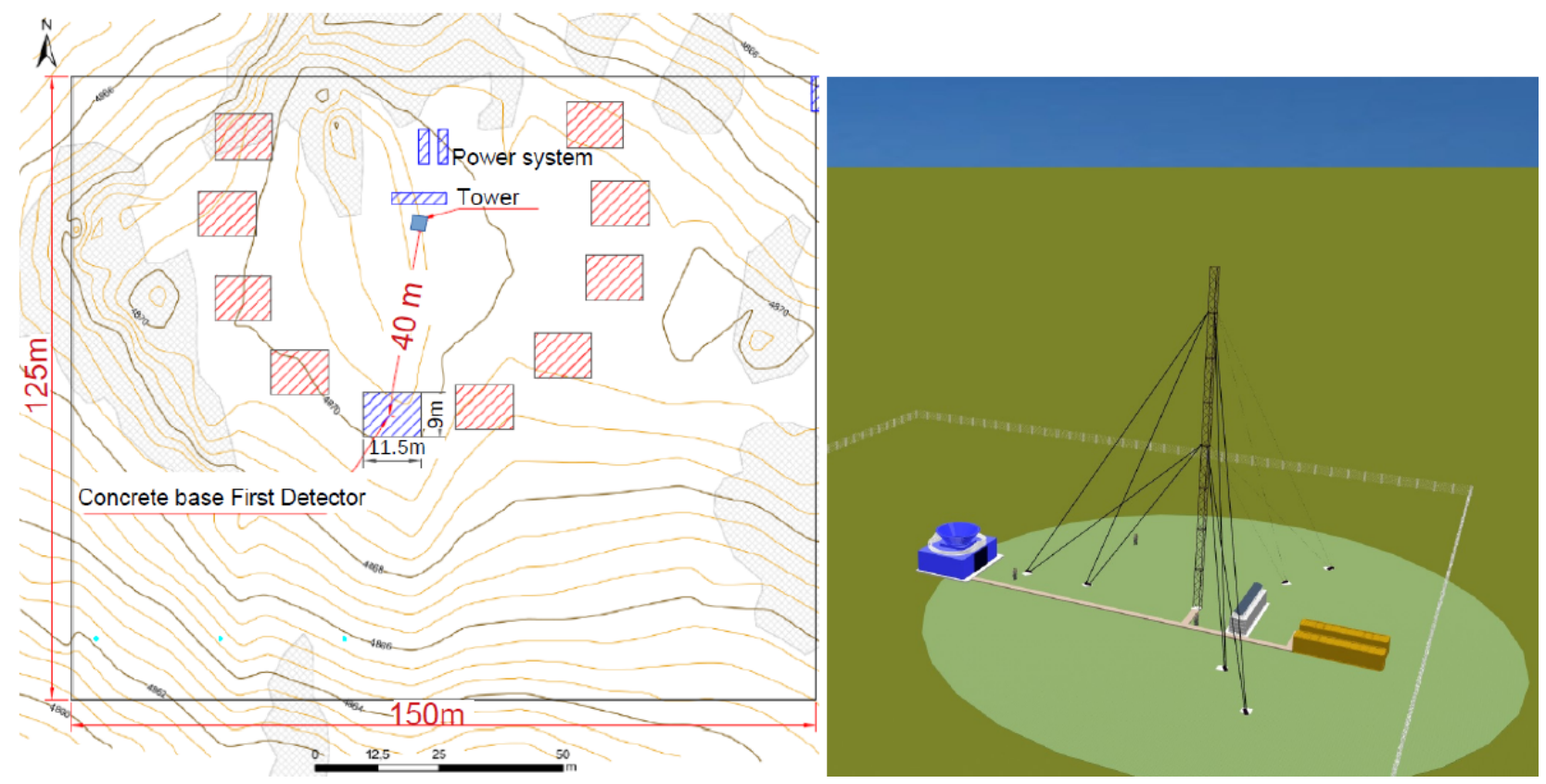

Figure 7. Left: detailed map of the area allocated to QUBIC. Slashed in blue the locations for the first installations, in red those reserved for future modules. Right: simulation of an aerial view for the first installations at the site, including the shelter with the first module and the tower for the calibration source.

The predominant climate in the area chosen for installation of the experiment is semi-arid continental, with significant fluctuations in temperature between day and night. The minimum temperature recorded was $-16^{\circ} \mathrm{C}$ with a maximum of $35^{\circ} \mathrm{C}$. Summer is a rainy season, but the rain is scarce (the annual average is $70-120 \mathrm{~mm}$ ). The snowfalls are also scarce and they only whiten the tops of the mountains.

Emission from atmospheric water vapour in the site is the main source of photon noise measured by background-limited detectors such as those in QUBIC. During the last decade, the Argentine Institute of Radio Astronomy (IAR), which leads the LLAMA project in Argentina, has continuously carried out several campaigns to obtain weather measurements in Alto Chorrillos in order to establish the general climatic characteristics of the site and in particular to measure its atmospheric opacity. These measurements were performed using a tipping radiometer operating at $210 \mathrm{GHz}$, instrument lent by the National Autonomous University of Mexico. The results obtained from these observations and the permanent monitoring of opacity, show that the site is within the specifications required by LLAMA at the frequency of $210 \mathrm{GHz}$ [46]. The measurements are summarized in the left panel of figure 8 . These and further measurements of opacity at 210
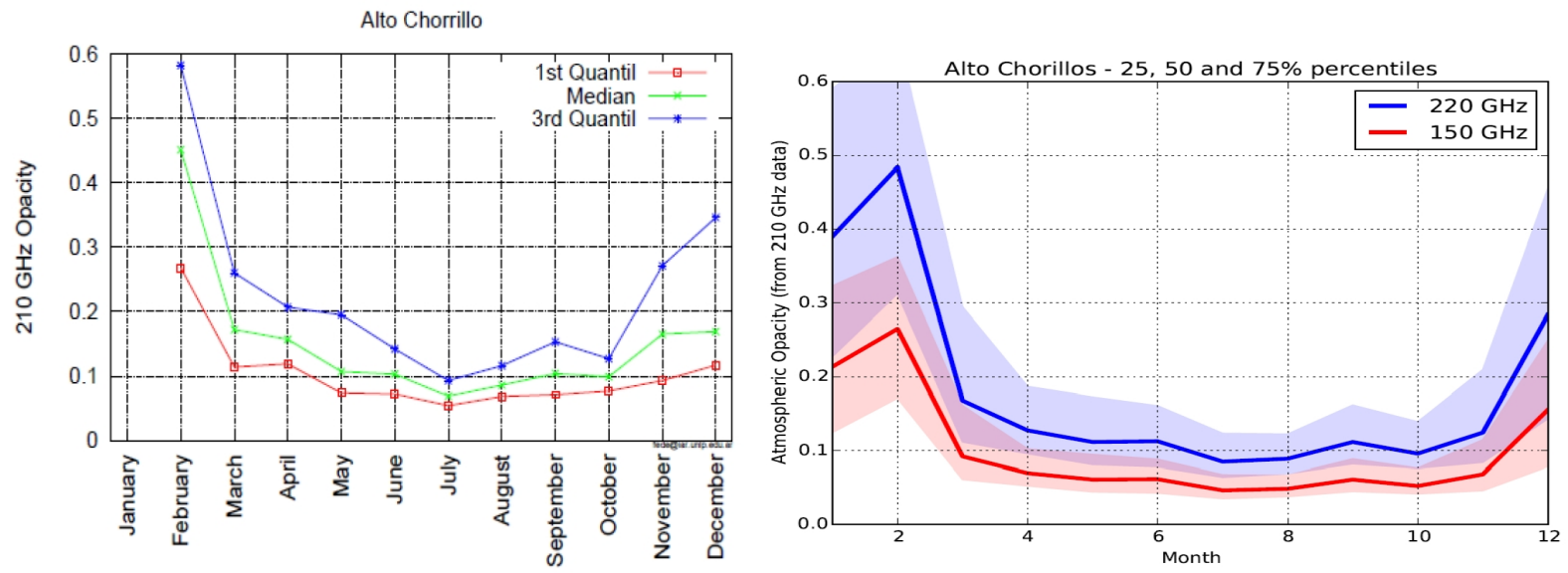

Figure 8. Left: atmospheric opacity at $210 \mathrm{GHz}$ as measured in Alto Chorrillos for each month (reproduced from [46]). Right: extrapolations to $150 \mathrm{GHz}$ and $220 \mathrm{GHz}$ using an atmospheric model for Chajnantor (reproduced from [27]). 
$\mathrm{GHz}$ (the published ones did not include January) were extrapolated to the frequencies at which QUBIC operates using an atmospheric emission spectrum model. Due to the proximity and similar geographical and geological characteristics, the Atmospheric Transmission at Microwaves (ATM) code [47] optimized for the atmospheric conditions in Chajnantor was used [27]. This is the site in Chile for the ALMA radio-telescopes, as well as for several other millimeter and submillimeter telescopes. The results of the extrapolation are shown in the right panel of figure 8. As we can see the atmospheric opacity is as good as $\tau<0.20$ for all months, except for January and February (part of what is known as the "Bolivian winter"). The values of atmospheric opacities are comparable to the results for Chajnantor.

\subsubsection{Weather measurements on site}

In addition to the measurements of atmospheric opacity described above, meteorological data was collected in the context of the LLAMA project using a standard station installed in May 2010, provided by researchers from the Institute of Astronomy, Geophysics and Atmospheric Sciences of Brazil, in order to monitor the atmospheric temperature, humidity, pressure, and wind speed and direction. All the meteorological variables collected for LLAMA are within the ranges for smooth operation of QUBIC: temperature between $-15^{\circ} \mathrm{C}$ and $+15^{\circ} \mathrm{C} 90 \%$ of the time, wind speed with a maximum over 1 minute less that $20 \mathrm{~m} / \mathrm{s}$, clear skies $90 \%$ of the time and atmospheric opacity $\tau<0.10$ (median) at $150 \mathrm{GHz}$ and $\tau<0.15$ (median) at $220 \mathrm{GHz}$.

Moreover, a new weather station was installed by the QUBIC collaboration in 2018, in order to record current data specifically at the QUBIC site. The ground data acquisition was performed using a DAVIS Vantage Pro II weather station (https://www.davisinstruments.com/solution/vantage-pro2/), located 3 meters above ground level and connected to a single board computer (SBC) and a data logger. The data were stored at the device in a convenient ad-hoc format, usually plain text. A simple database was designed to store all data in a consistent way, also providing a quick way to choose and process the data. The data were acquired every minute, all year round, and averaged every 10 minutes before the analysis. Figure 9 presents the results from the ground-based weather station in Alto Chorrillos. The average temperature and its dispersion
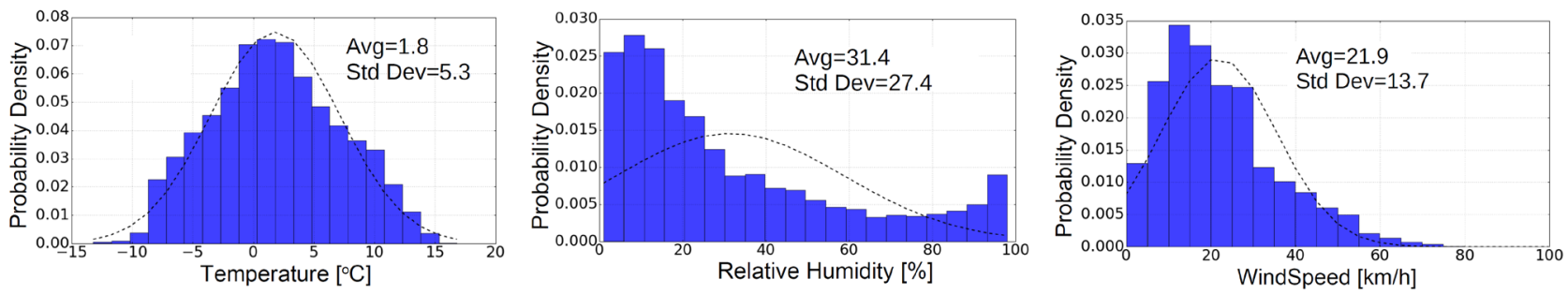

Figure 9. Histograms for the probability density of ambient temperature (left), humidity (center) and wind speed (right) at the Alto Chorrillos site. A normal distribution with the average and dispersion as in data is also plotted (dashed lines) for reference.

are $(1.8 \pm 5.3)^{\circ} \mathrm{C}$. The daily probability density for relative humidity is $31.4 \%$ in average with a standard deviation of about $\pm 27.5 \%$. The analysis was performed for $24 \mathrm{hs}$. of data. The wind speed average is $21.9 \mathrm{~km} / \mathrm{h}(6.1 \mathrm{~m} / \mathrm{s})$, with a standard deviation of $\pm 13.7 \mathrm{~km} / \mathrm{h}$. The wind gusts are, in general, a big restriction for astronomical facilities. In the case of the site chosen for QUBIC, the wind presents maximum speeds of $100 \mathrm{~km} / \mathrm{h}(28 \mathrm{~m} / \mathrm{s})$. This is fundamental data to estimate the resistance needed by the shelter and the dome for the instrument.
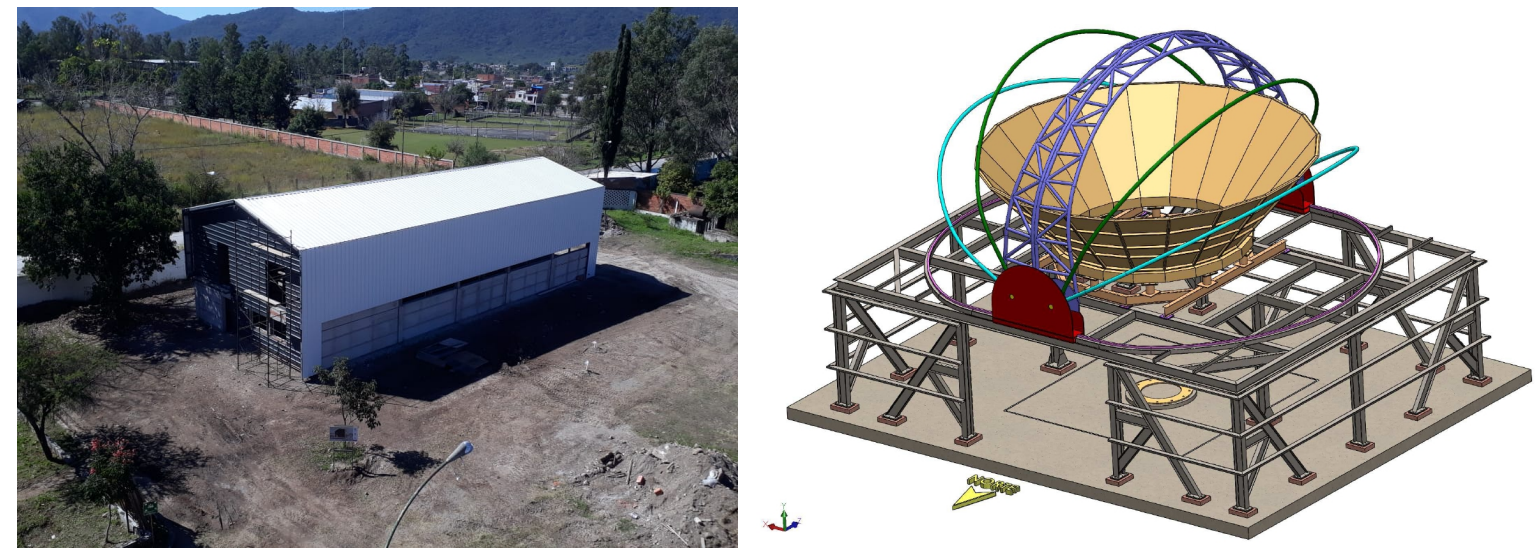

Figure 10. Ongoing construction of the Integration Laboratory in Salta City as of May 2019 (left) and design of the structure for shelter, dome and ground-shield for QUBIC (right). 


\subsubsection{Site development and infrastructure}

Several developments related to the site are ongoing, mainly devoted to preparations to test the instrument upon arrival to Argentina and to secure its installation in Alto Chorrillos. The access road to the LLAMA site and from there to the QUBIC site has already been built, and constructions towards the installation of the first module are under way.

Figure 10 shows in its left panel the Integration Laboratory under construction in Salta city, the place where the instrument will be integrated and tested prior to installation in its final site, and the final design for the shelter and the dome for the instrument in its right panel. An open-foldable dome with high-tension textile membranes was chosen for the cover in order to reduce weight. The weather conditions, mainly the wind speed, were accounted for in the simulations of resistance of the complete building. The construction of the shelter is planned to be completed before the end of 2019 .

\subsection{Detection and analysis chain}

\subsubsection{Signal model and synthesized beam}

The image that QUBIC will observe in each of the two focal planes is the result of an interferometric pattern that arises from the superposition of the signals of each of the secondary antennas in the horn array. Due to the time modulation introduced by the rotating half-wave plate (HWP), the signal measured by each of the bolometric detectors $p$ in the focal plane with frequency $v$ and at time $t$ can be described by:

$$
R(p, v, t)=K\left[S_{I}(p, v)+\cos \left(4 \phi_{H W P}(t)\right) S_{Q}(p, v)+\sin \left(4 \phi_{H W P}(t)\right) S_{U}(p, v)\right]
$$

where $\phi_{H W P}(t)$ is the angle of the HWP at time $t$, and $K$ is an overall calibration constant that takes into account the efficiency of the optical chain. The three terms $S_{I, Q, U}$ in Equation 8 represent the sky signal in intensity and polarization convolved with the so-called synthesized beam (see Eq. 10).

As more and more horns are open in the observing array, the number of baselines increases (a baseline is the vector separating two horns). The constructive and destructive superposition of the re-emitted signals produces an effective beam which is highly non-trivial. In fact, the synthesized beam of QUBIC has a complicated shape, with a central peak and replications. The beam can be approximated by a central gaussian with secondary gaussian peaks, separated by an angular distance of about $8.5^{\circ}$ with a width equal to the full-width at half-maximum (FWHM) at the observing frequency. Both the separation distance between peaks and their width depend on the frequency of the received signal. In the left panel of figure 11, a schematic plot of the synthesized beam for two monochromatic frequencies is shown, where it is clear the dependence on the observing frequency of the separation between primary and secondary peaks. The amplitudes of the primary and secondary peaks are modulated by a gaussian which is equal to the primary beam of the input horn. For a finite bandwidth, the beam is computed as the superposition of all the monochromatic beams included in the band, and due to the dependence on the frequency, the secondary peaks appear asymmetric and elongated. In the right panel of Figure 11 we show the synthesized beam of the central pixel, considering a $30 \%$ bandwidth.

\subsubsection{Point source imaging}

Taking into account all of the detectors in the focal plane, we can study the image obtained for a far-away point source located along the line of sight. The images in figure 12 shows the resulting synthesized beam on the focal plane. When QUBIC observes a far point-source located along the line-of-sight, with all 400 antennas open, the image formed in the focal planes is an interference pattern with the shape of the synthesized beam. It consists of peaks and lobes, and it is regarded as the beam that will convolve the sky-signal at observation time.

More specifically, if $X$ is the signal from the sky (either in intensity, $I$, or polarization, $Q, U$ ), then the measured signal on the pixel $p$ is $S_{X}(p)=\int X(\mathbf{n}) B_{\text {synth }}^{p}(\mathbf{n}) d \mathbf{n}$. This means that QUBIC data can be analyzed similarly to the data obtained from a normal imager, provided that we build a window function of the synthesized pattern for each pixel. The synthesized beam $B_{\text {synth }}^{p}(\mathbf{n})$ is obtained through the interference of the beams of different horns, and can be described, in the absence of optical aberrations, by

$$
B_{\text {synth }}^{p}(\mathbf{n}, \mathbf{r}, \lambda)=\left|\sum_{i} B_{\text {prim }}(\mathbf{n}) B_{\text {sec }}(\mathbf{r}) \exp \left(i 2 \pi \frac{\mathbf{x}_{i}}{\lambda}\left(\frac{\mathbf{r}}{D_{f}}-\mathbf{n}\right)\right)\right|^{2}
$$

where $B_{\text {prim }}^{p}(\mathbf{n})$ is the primary beam (the input beam for horns pointing to the sky at direction $\left.\mathbf{n}\right) ; B_{\text {sec }}(\mathbf{r})$ is the secondary beam acting at the $\mathbf{r}$ point in the focal plane (i.e. in the pixel $p$ ), $\mathbf{x}_{i}$ is the position of the horn $i ; D_{f}$ is the focal distance. For a square horn array the sum in the last equation can be computed analytically to give

$$
B_{\text {synth }}^{p}(\mathbf{n}, \mathbf{r}, \lambda)=B_{\text {prim }}(\mathbf{n}) B_{\text {sec }}(\mathbf{r}) \frac{\sin ^{2}\left[n_{h} \pi \frac{\Delta x}{\lambda}\left(\frac{r_{x}}{D_{f}}-n_{x}\right)\right] \sin ^{2}\left[n_{h} \pi \frac{\Delta x}{\lambda}\left(\frac{r_{y}}{D_{f}}-n_{y}\right)\right]}{\sin ^{2}\left[\pi \frac{\Delta x}{\lambda}\left(\frac{r_{x}}{D_{f}}-n_{x}\right)\right] \sin ^{2}\left[\pi \frac{\Delta x}{\lambda}\left(\frac{r_{y}}{D_{f}}-n_{y}\right)\right]}
$$



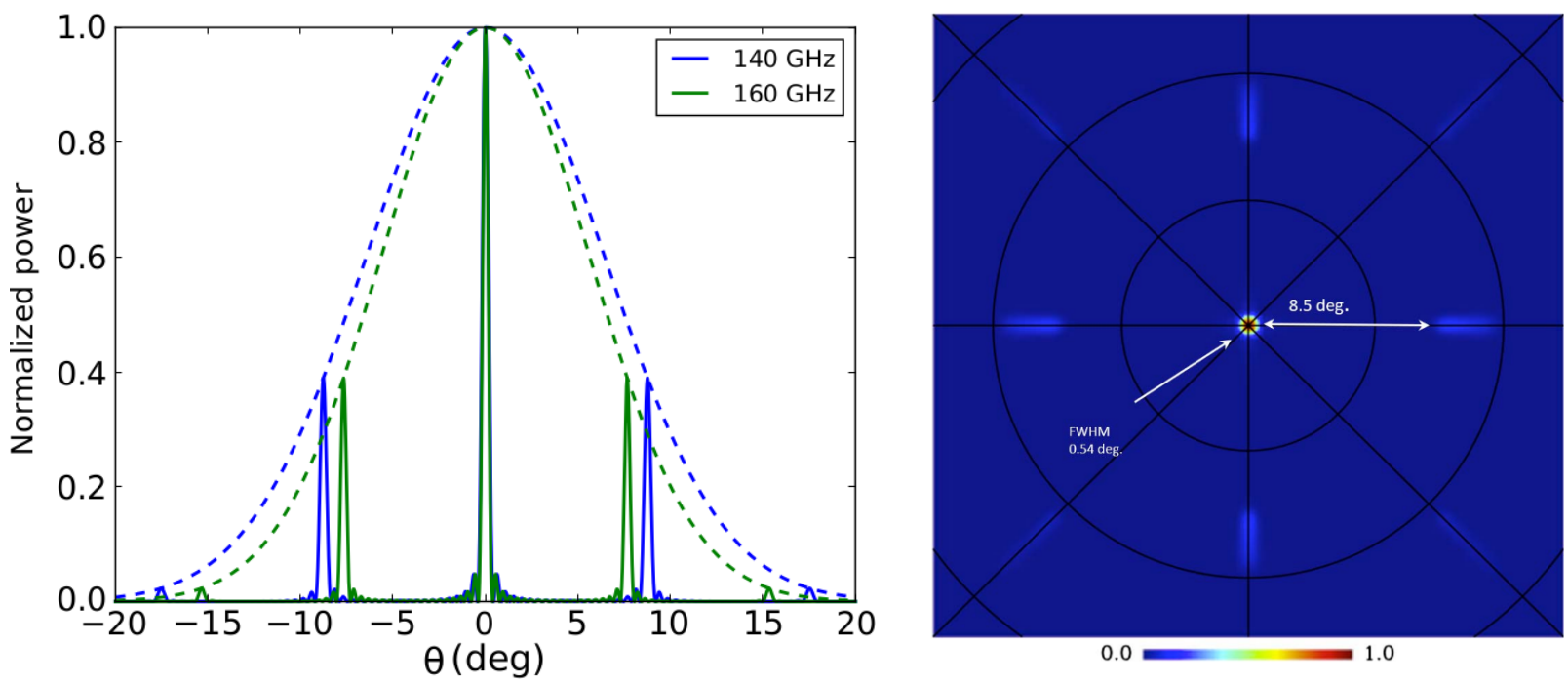

Figure 11. Left panel: Synthesized beam for the monochromatic case, for two nearby frequencies. The separation between the primary and secondary peaks of the beam depends on the frequency of the signal. Right panel: Simulation of the synthesized beam of the center pixel of the $150 \mathrm{GHz}$ array, taking into account the finite (30\%) bandwidth of the detectors. The addition of all of the secondary peaks of the monochromatic beams included in the band results on secondary peaks which are asymmetric and elongated. Figure adapted from [24] and [28].

where $n_{h}$ is the number of horns on one side of the square horn array and $\Delta x$ is the distance between them [24].

\subsubsection{Map-making}

QUBIC will observe a partial area of the sky, similar to the region observed by the BICEP2 Collaboration (another observational effort to measure the primordial B-modes [37]). It is a region with relatively low galactic foregrounds, although they cannot be neglected. This is the main reason why the instrument has two frequency bands, and the relevance of the spectro-imaging properties of QUBIC, which will be described in subsection 2.4.2. The contamination from foregrounds can be alleviated when the sky is observed in several frequencies.

When the signal is not a point-source but an extended region in the sky, as the QUBIC area will be, every detector in the focal plane will receive the signal from different directions in the sky, due to the non-trivial shape of the synthesized beam.

Exactly in the same way as for a usual imager, we can scan the sky with our synthesized beam with any scanning strategy, and any individual measurement results in the convolution of the sky through our synthesized beam. From the Time-Ordered Data (TOD) of each individual bolometer, we can reconstruct a map of the sky, taking into account the special shape of the synthesized beam.

\subsection{Calibration and expected performance}

\subsubsection{Self-calibration}

The design of QUBIC as a bolometric interferometer allows the exploitation of redundant interferometric patterns to control and reduce the systematics of the experiment. By using different combinations of open horns in the array, we can obtain a number of redundant baselines that can be used to self-calibrate the instrument. This technique is derived from radio-interferometry self-calibration [48].

To understand the basic concept of self-calibration, it is important to note that the interference pattern produced by two open horns will be exactly the same as one obtained with a different pair of open horns but defining exactly the same baseline (separation and orientation of the pair of open horns). For an ideal instrument, with no systematic effects, the interferometric pattern depends only on the baseline. Hence, we can use the observations obtained with equivalent baselines to account for the systematics of the instrument, by analysing the differences observed with different but equivalent baselines.

The theoretical framework of the self-calibration procedure is described in detail in [49]. The instrumental systematics are modelled via the use of Jones-matrices, which are used in cascade to describe the action of an optical chain onto the incoming radiation electric field (attenuation, polarization mixing, dephasing). For an instrument with several components, there is a Jones-matrix for each of them. In this way, the effect of each part of the instrument can be parametrized with a given model.

Systematic errors can come in the form of horn location errors, pointing errors, asymmetries of beams, bolometer location errors, diagonal and non-diagonal terms of the Jones-matrices, and so forth. Due to the time modulation of the polarized signal given by the HWP (eq. 8), and the subsequent action of the interference array, for a real instrument, there is 

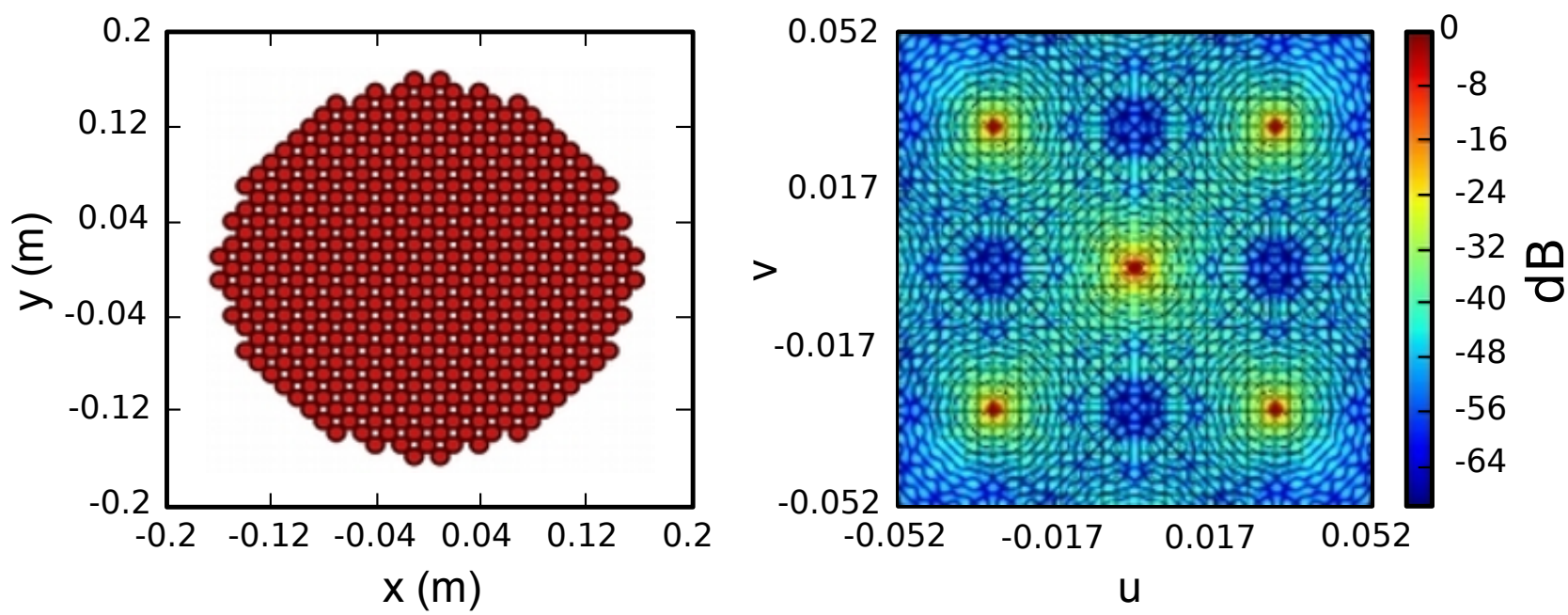

Figure 12. Left: QUBIC aperture plane showing all 400 antennas open to the sky. Right: the interference pattern formed on each of the focal planes when the instrument is observing a point-source located in the far field vertically along the instrument line-of-sight. The $\mathrm{u}$ and $\mathrm{v}$ coordinates are defined as: $\mathrm{u}=\sin \theta \cos \phi$ and $\mathrm{v}=\sin \theta \sin \phi$, where $\theta$ and $\phi$ are the angles on the celestial sphere defining the synthesized beam. Figure reproduced from [28].

the possibility of a leakage from $\mathrm{Q}$ to $\mathrm{U}$ and vice versa, due to uncertainties and errors in the process. Therefore, there will be a leakage between the $E$ and $B$-modes, in particular from $E$ to $B$ (the original $B$-mode is expected to be much lower than the $E$-mode, hence the leakage from $B$ to $E$ might be neglected). We can further give a constraint to the induced value of the tensor-to-scalar ratio $r$. The leakage from $E$-modes to $B$-modes is significantly reduced by applying the self-calibration, even spending only $1 \mathrm{sec}$ per baseline in the procedure. The leakage can be further reduced by spending more time on self-calibration. During the self-calibration phase, pairs of horns are successively shut while QUBIC observes an artificial partially-polarized source (a microwave synthesizer) in the far field. Then, the signal measured by each individual pair of horns is reconstructed and compared with each other. If the source is stable, then redundant baselines correspond to the same mode of the observed field, and therefore, a different signal between them can only be due to photon noise or instrumental systematic effects. Using a detailed parametric model of the instrument, we can fully recover the instrument parameters. The updated model of the instrument can then be used to reconstruct the synthesized beam and improve the map-making, thus reducing the leakage from $E$ to $B$-modes.

In Figure 13 we show the improvement in the power spectrum estimation with self-calibration, depending on the time spent in the calibration mode. The more time spent in self-calibration mode, the lower the $E$ to $B$ leakage due to instrumental imperfections. In black solid lines, the prediction of the $B$-mode polarization power spectrum is displayed, for different values of the tensor-to-scalar ratio $r$.

\subsubsection{Spectral imaging}

The particular shape of the synthesized beam of the QUBIC experiment enables a peculiar and most convenient use of the instrument: that of a spectro-imager. Indeed, the dependence of the angular distance between primary and secondary peaks of the beam on the frequency of the observed signal allows the separation of the signal into different subfrequencies inside each channnel, providing information at more frequency bands than originally designed for. In this way, we can count on more frequency channels to disentangle the CMB polarization signal from those of polarized galactic foregrounds.

\section{Current status and forecasts}

The QUBIC instrument is currently undergoing its phase of laboratory calibration of its technological demonstrator version. A single focal plane with 256 TES detectors operating at $150 \mathrm{GHz}$ and an array of 64 back-to-back horns along with a smaller optical combiner have been integrated into the final $1 \mathrm{~K}$ box and cryostat. The horns array include the individual switches that enable the auto-calibration procedure. The cryogenic system has been succesfully cycled and tested to reach the expected performance.

An image of the synthesized beam at various frequencies has been successfully measured in several bolometers in the focal plane of the technological demonstrator, using the laboratory calibration source. As an example, in Fig. 14 we show a comparison between the predicted synthesized beam at $150 \mathrm{GHz}$ and the one measured in one of the TES. The left panel displays the measurement, and the right panel shows the expected shape without aberrations (geometrical optics). The signal is normalized to the maximum value in each of the maps. There is some degree of saturation in the simulated image, to mimic what is observed in the measurement. Albeit only qualitative, the agreement shown here constitutes good evidence of the successful performance of the instrument design. A quantitative characterization of the response of all 


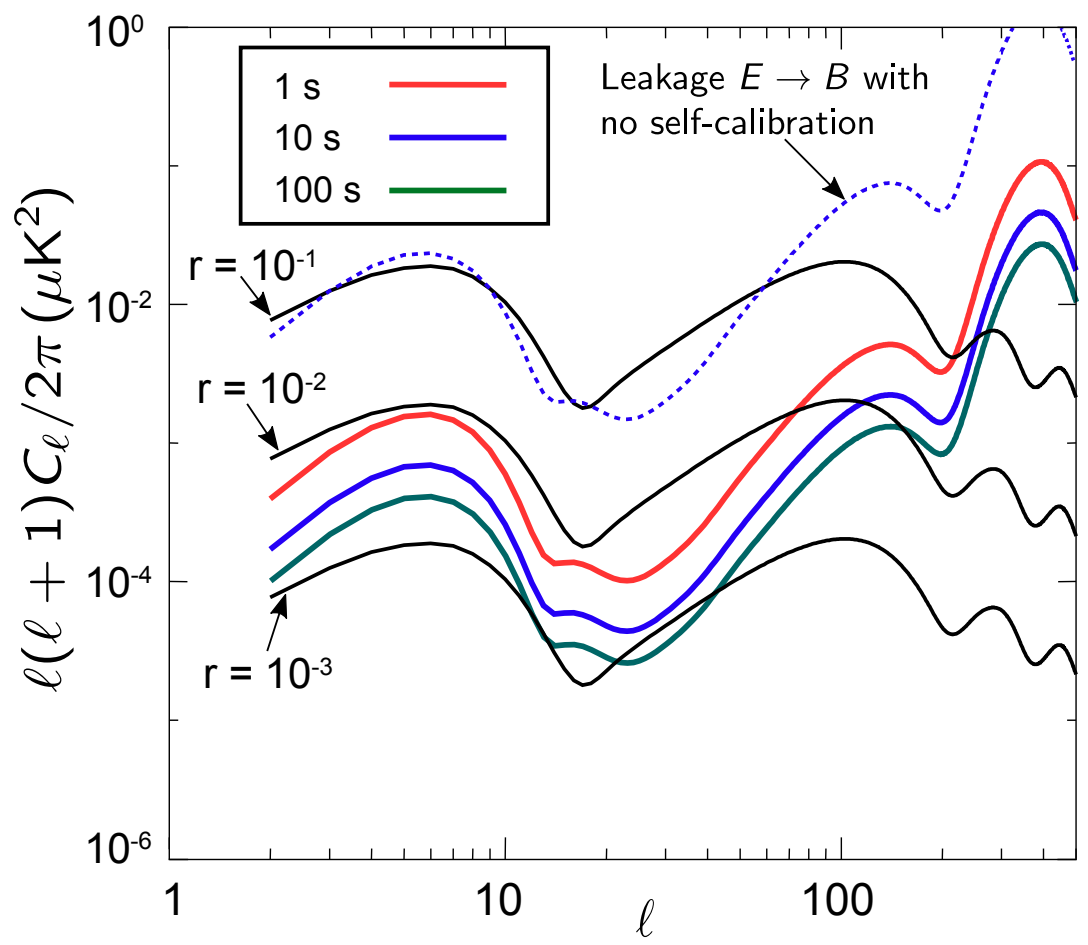

Figure 13. Improvement in the recovery of the $B$-mode power spectrum as a function of the time spent in the self-calibration mode. The three curves drawn with black solid lines represent theoretical B-mode power spectra calculated for three different values of the tensor-to-scalar ratio $r$. Figure reproduced from [28], adapted from [49].

detectors will be reported elsewhere, after the calibration of the technological demonstrator is completed. More details on the simulation of the optical beam combiner and horns array can be found in [50].

Furthermore, the angular resolution of the spectro-imager has been computed, and follows what is expected for the different subfrequency bands, namely, that the FWHM is reduced with increasing frequency.

After calibration is completed the TD will be shipped to Argentina for a first-light test on site in Alto Chorrillos. Meanwhile construction of the two complete focal panels at $150 \mathrm{GHz}$ and $220 \mathrm{GHz}$, the full horns array and the final optical combiner will proceed. It is expected that the first module of the QUBIC instrument will be ready by 2020.

With two years of operation of its first module and an efficiency of 30\% QUBIC is expected to be sensitive to values of the tensor-to-scalar ratio parameter $r$ as low as 0.01. In Table 1 we summarise the expected sensitivity of QUBIC and other major ground-based experiments in the same frequency range, either running or expected to be deployed in the near future. It is important to notice that not only the reachable sensitivity should be taken into account, but also the systematic effects control and the possibility to remove foregrounds. In this sense, QUBIC has a unique status due to its particular architecture as a bolometric interferometer.

\begin{tabular}{cccccc}
\hline Project & Frequencies $(\mathrm{GHz})$ & $\ell$ range & Ref. & $\sigma(r)$ goal (no fg.) & $\sigma(r)$ goal (with fg.) \\
\hline QUBIC & 150,220 & $30-200$ & & $6.0 \times 10^{-3}$ & $1.0 \times 10^{-2}$ \\
Bicep3/Keck & $95,150,220$ & $50-250$ & {$[18]$} & $2.5 \times 10^{-3}$ & $1.3 \times 10^{-2}$ \\
CLASS ${ }^{\dagger}$ & $38,93,148,217$ & $2-100$ & {$[20]$} & $1.4 \times 10^{-3}$ & $3.0 \times 10^{-3}$ \\
SPT-3G $^{\dagger}$ & $95,148,223$ & $50-3000$ & {$[19]$} & $1.7 \times 10^{-3}$ & $5.0 \times 10^{-3}$ \\
AdvACT & $90,150,230$ & $60-3000$ & {$[21]$} & $1.3 \times 10^{-3}$ & $4.0 \times 10^{-3}$ \\
Simons Array & $90,150,220$ & $30-3000$ & {$[22]$} & $1.6 \times 10^{-3}$ & $5.0 \times 10^{-3}$ \\
Simons Observatory (SAT) $^{\star \star}$ & $27,39,93,145,225,280$ & $30-300$ & {$[23]$} & $1.3 \times 10^{-3}$ & $3.9 \times 10^{-3}$ \\
\hline
\end{tabular}

${ }^{\star}$ CLASS: Cosmology Large Angular Scale Surveyor; ${ }^{\dagger}$ SPT-3G: South Pole Telescope—3rd generation; ${ }^{\star}$ AdvACT: Advanced Atacama Cosmology Telescope; ${ }^{\star \star}$ SAT: Small Aperture Telescopes.

Table 1. Sensitivity of the main $B$-mode ground experiments operating in a frequency range similar to QUBIC. The label "fg" or "no fg" corresponds to the assumption on the foregrounds. Table extracted and expanded from [27, 28]. The entries for $\sigma(r)$ correspond to the estimates made in [40] for different experiments with the algorithms developed there (Appendix B1, no-delensing option). We acknowledge use of the public code CMB4CAST (https://portal.nersc.gov/project/mp107) for the computation of the last entry with the same method as in [40]. 

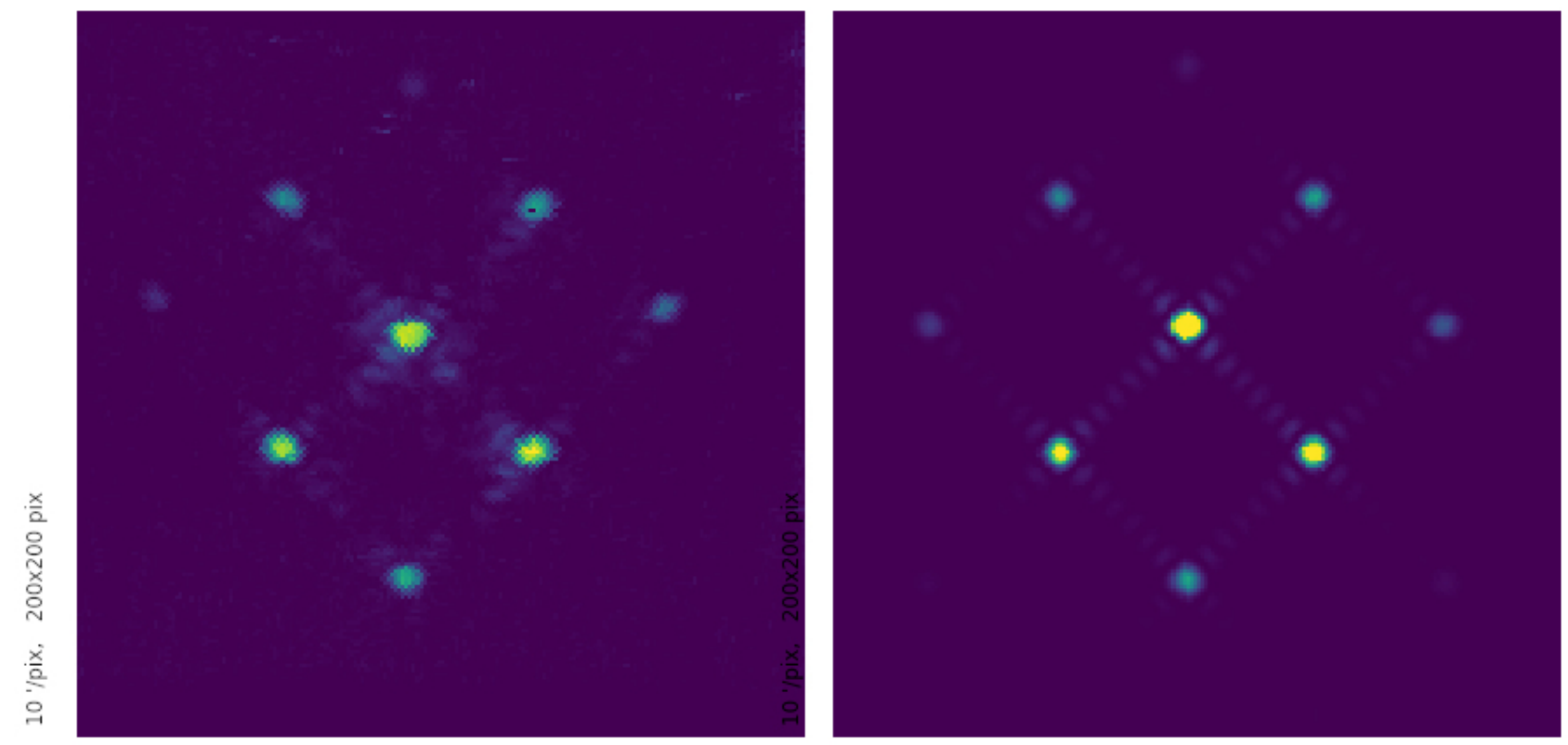

Figure 14. Comparison between the synthesised beam measured with one of the TES in the technological demonstrator (left panel) and the predicted beam without aberration effects (right panel).

In the future, more modules at additional frequencies could be added, aiming at improving the foreground removal, and increasing the sensitivity to the primordial $B$-mode polarization of the CMB.

\section{Conclusions}

The CMB provides a way to indirectly probe for the presence of primordial gravitational waves with cosmological wavelengths, with the size of the observable universe. Gravitational waves would have imprinted upon the CMB a specific pattern of $B$-mode polarization at the time the relic photons decoupled from matter almost 14 billion years ago. The quest for the detection of primordial $B$-modes in the CMB is a major experimental challenge, which is being pursued by several experiments, and up to now resulted in an upper bound on the tensor-to-scalar ratio $r<0.06$ at $95 \%$ confidence level. The detection of primordial $B$-modes and the determination of the parameter $r$ would be a major milestone in modern cosmology. It would test inflationary cosmological models, that predict that quantum effects at the earliest stages of the Big-Bang produced gravitational waves along with the density fluctuations that later seeded galaxy formation, but do not make definite predictions for their relative intensity.

QUBIC will soon join the existing efforts to search for primordial $B$-modes. It will do it from the Puna in Salta, Argentina, with a novel kind of instrument. With its unique architecture as a bolometric interferometer, it will benefit from the excellent sensitivity of the TES detectors, as well as from the high levels in the control of systematic effects provided by the self-calibration procedure based on the comparison of redundant baselines. Interferometric operation will also allow for spectral-imaging, which will improve the subtraction of astrophysical foregrounds with multi-frequency measurements.

After calibration is completed, QUBIC “Technological Demonstrator" will be shipped to Argentina for installation in Alto Chorrillos, while construction of the complete first module proceeds. In two years of operation of the complete instrument values of the tensor-to-scalar ratio $r$ of order 0.01 will be probed with a method alternative to those of other current efforts. The successful performance of QUBIC will open a new road in the search of the elusive primordial $B$-modes of CMB polarization, footprints of gravitational waves from an inflationary period during the earliest moments in the history of the Universe.

\section{Acknowledgments}

QUBIC is funded by the following agencies. France: ANR (Agence Nationale de la Recherche) 2012 and 2014, DIMACAV (Domaine d'Interet Majeur-Astronomie et Conditions d'Apparition de la Vie), CNRS/IN2P3 (Centre national de la recherche scientifique/Institut national de physique nucléaire et de physique des particules), CNRS/INSU (Centre national de la recherche scientifique/Institut national de sciences de l'univers). Maria Salatino acknowledges the financial support of the UnivEarthS Labex program at Sorbonne Paris Cité (ANR-10-LABX-0023 and ANR-11-IDEX-0005-02. 
Italy: CNR/PNRA (Consiglio Nazionale delle Ricerche/Programma Nazionale Ricerche in Antartide) until 2016, INFN (Istituto Nazionale di Fisica Nucleare) since 2017. Argentina: Secretaría de Gobierno de Ciencia, Tecnología e Innovación Productiva, Comisión Nacional de Energía Atómica, Consejo Nacional de Investigaciones Científicas y Técnicas. UK: the University of Manchester team acknowledges the support of STFC (Science and Technology Facilities Council) grant ST/L000768/1. Ireland: James Murphy and David Burke acknowledge postgraduate scholarships from the Irish Research Council. Duc Hoang Thuong acknowledges the Vietnamese government for funding his scholarship at APC. Andrew May acknowledges the support of an STFC PhD Studentship.

\section{References}

[1] A. A. Penzias and R. W. Wilson. A Measurement of Excess Antenna Temperature at 4080 Mc/s. ApJ, 142:419-421, July 1965.

[2] G. F. Smoot, C. L. Bennett, A. Kogut, E. L. Wright et al. Structure in the COBE differential microwave radiometer first-year maps. ApJL, 396:L1-L5, September 1992.

[3] Planck Collaboration: N. Aghanim, Y. Akrami, M. Ashdown, J. Aumont et al. Planck 2018 results. VI. Cosmological parameters. arXiv eprints 1807.06209, July 2018.

[4] M. J. Rees. Polarization and Spectrum of the Primeval Radiation in an Anisotropic Universe. ApJL, 153:L1, July 1968.

[5] J. M. Kovac, E. M. Leitch, C. Pryke, J. E. Carlstrom, N. W. Halverson, and W. L. Holzapfel. Detection of polarization in the cosmic microwave background using DASI. Nature, 420:772-787, December 2002.

[6] A. G. Polnarev. Polarization and Anisotropy Induced in the Microwave Background by Cosmological Gravitational Waves. Soviet Astronomy, 29:607-613, December 1985.

[7] R. Crittenden, R. L. Davis, and P. J. Steinhardt. Polarization of the Microwave Background Due to Primordial Gravitational Waves. ApJL, 417:L13, November 1993.

[8] D. D. Harari and M. Zaldarriaga. Polarization of the microwave background in inflationary cosmology. Physics Letters B, 319:96-103, December 1993.

[9] U. Seljak and M. Zaldarriaga. Signature of Gravity Waves in the Polarization of the Microwave Background. Physical Review Letters, 78:2054-2057, March 1997.

[10] M. Kamionkowski, A. Kosowsky, and A. Stebbins. A Probe of Primordial Gravity Waves and Vorticity. Physical Review Letters, 78:2058-2061, March 1997.

[11] D. Hanson, S. Hoover, A. Crites et al. Detection of B-Mode Polarization in the Cosmic Microwave Background with Data from the South Pole Telescope. Physical Review Letters, 111(14):141301, October 2013.

[12] The Polarbear Collaboration. A Measurement of the Cosmic Microwave Background B-mode Polarization Power Spectrum at Sub-degree Scales with POLARBEAR. ApJ, 794:171, October 2014.

[13] M. Zaldarriaga and U. Seljak. Gravitational lensing effect on cosmic microwave background polarization. Physical Review D, 58(2):023003, July 1998.

[14] B.P. Abbott et al. (LIGO Scientific Collaboration and Virgo Collaboration. Observation of gravitational waves from a binary black hole merger. Phys. Rev. Lett., 116:061102, Feb 2016.

[15] A. H. Guth. Inflationary universe: A possible solution to the horizon and flatness problems. Physical Review D, 23:347-356, January 1981.

[16] A. D. Linde. A new inflationary universe scenario: A possible solution of the horizon, flatness, homogeneity, isotropy and primordial monopole problems. Physics Letters B, 108:389-393, February 1982.

[17] A. Albrecht and P. J. Steinhardt. Cosmology for grand unified theories with radiatively induced symmetry breaking. Physical Review Letters, 48:1220-1223, April 1982.

${ }^{[18]}$ H. Hui, P. A. R. Ade, Z. Ahmed, K. D. Alexander, M. Amiri, D. Barkats, S. J. Benton, C. A. Bischoff, J. J. Bock et al. BICEP3 focal plane design and detector performance. In Millimeter, Submillimeter, and Far-Infrared Detectors and Instrumentation for Astronomy VIII, volume 9914 of Society of Photo-Optical Instrumentation Engineers (SPIE) Conference Series, page 99140T, Jul 2016.

[19] B. A. Benson, P. A. R. Ade, Z. Ahmed, S. W. Allen, K. Arnold, J. E. Austermann, A. N. Bender, L. E. Bleem et al. SPT-3G: a next-generation cosmic microwave background polarization experiment on the South Pole telescope. In Millimeter, Submillimeter, and Far-Infrared Detectors and Instrumentation for Astronomy VII, volume 9153 of Society of Photo-Optical Instrumentation Engineers (SPIE) Conference Series, page 91531P, Jul 2014. 
[20] K. Harrington, T. Marriage, A. Ali, J. W. Appel, C. L. Bennett, F. Boone, M. Brewer, M. Chan et al. The Cosmology Large Angular Scale Surveyor. In Millimeter, Submillimeter, and Far-Infrared Detectors and Instrumentation for Astronomy VIII, volume 9914 of Society of Photo-Optical Instrumentation Engineers (SPIE) Conference Series, page 99141K, Jul 2016.

[21] S. W. Henderson et al. Advanced ACTPol Cryogenic Detector Arrays and Readout. J. Low. Temp. Phys., 184(3-4):772779, 2016.

${ }^{[22]}$ K. Arnold, N. Stebor, P. A. R. Ade, Y. Akiba, A. E. Anthony et al. The Simons Array: expanding POLARBEAR to three multi-chroic telescopes. In Millimeter, Submillimeter, and Far-Infrared Detectors and Instrumentation for Astronomy VII, volume 9153 of Proc. SPIE, page 91531F, August 2014.

[23] P. Ade, J. Aguirre, Z. Ahmed, S. Aiola, A. Ali et al. The Simons Observatory: science goals and forecasts. Journal of Cosmology and Astro-Particle Physics, 2019(2):056, Feb 2019.

[24] QUBIC Collaboration: E. Battistelli, A. Baú, D. Bennett, L. Bergé, J. P. Bernard, P. de Bernardis, G. Bordier, A. Bounab et al. QUBIC: The QU bolometric interferometer for cosmology. Astroparticle Physics, 34:705-716, April 2011.

[25] M. Piat, E. Battistelli, A. Baù et al. QUBIC: the Q\&U Bolometric Interferometer for Cosmology. J. Low. Temp. Phys., 167(5-6):872-878, 2012.

[26] A. Tartari, J. Aumont, S. Banfi, P. Battaglia, E. S. Battistelli, A. Baù, B. Bélier, D. Bennett, L. Bergé, J. P. Bernard et al. QUBIC: A Fizeau Interferometer Targeting Primordial B-Modes. J. Low. Temp. Phys., 184(3-4):739-745, 2016.

[27] J. Aumont, S. Banfi, P. Battaglia, E. S. Battistelli, A. Baù, B. Bélier, D. Bennett, L. Bergé et al. QUBIC Technical Design Report. arXiv:1609.04372, Sep 2016.

[28] A. Mennella, P. Ade, G. Amico, D. Auguste, J. Aumont, S. Banfi, G. Barbaràn, P. Battaglia, E. Battistelli, A. Baù et al. QUBIC: Exploring the Primordial Universe with the Q\&U Bolometric Interferometer. Universe, 5:42, January 2019.

[29] K.D. Irwin and G.C. Hilton. Transition-Edge Sensors, pages 63-150. in Cryogenic Particle Detection, C. Enss (editor), Springer, Berlin, Heidelberg, 2005.

[30] R. A. Hulse and J. H. Taylor. Discovery of a pulsar in a binary system. Astrophysics Journal Letters, 195:L51-L53, January 1975.

[31] D. N. Spergel and M. Zaldarriaga. Cosmic Microwave Background Polarization as a Direct Test of Inflation. Physical Review Letters, 79:2180-2183, September 1997.

[32] The BICEP/Keck Collaboration: P. A. R. Ade, Z. Ahmed, R. W. Aikin, K. D. Alexander, D. Barkats, S. J. Benton, C. A. Bischoff, J. J. Bock et al. Measurements of Degree-Scale B-mode Polarization with the BICEP/Keck Experiments at South Pole. arXiv:1807.02199, Jul 2018.

[33] M. Zaldarriaga and U. Seljak. All-sky analysis of polarization in the microwave background. Physical Review D, 55:1830-1840, February 1997.

[34] C. Scóccola, D. Harari, and S. Mollerach. B polarization of the CMB from Faraday rotation. Physical Review D, 70(6):063003, September 2004.

[35] S. Mollerach, D. Harari, and S. Matarrese. CMB polarization from secondary vector and tensor modes. Physical Review D, 69(6):063002, March 2004.

[36] David H. Lyth. What Would We Learn by Detecting a Gravitational Wave Signal in the Cosmic Microwave Background Anisotropy? Physical Review Letters, 78(10):1861-1863, Mar 1997.

[37] Bicep2 Collaboration. Detection of B-Mode Polarization at Degree Angular Scales by BICEP2. Physical Review Letters, 112(24):241101, June 2014.

[38] BICEP2/Keck Collaboration and Planck Collaboration: P. A. R. Ade, N. Aghanim, Z. Ahmed, R. W. Aikin, K. D. Alexander, M. Arnaud, J. Aumont, C. Baccigalupi et al. Joint Analysis of BICEP2/Keck Array and Planck Data. Physical Review Letters, 114(10):101301, March 2015.

[39] BICEP2 Collaboration and Keck Array Collaboration: P. A. R. Ade, Z. Ahmed, R. W. Aikin, K. D. Alexander et al. Constraints on Primordial Gravitational Waves Using Planck, WMAP, and New BICEP2/Keck Observations through the 2015 Season. Physical Review Letters, 121(22):221301, November 2018.

[40] J. Errard, S. M. Feeney, H. V. Peiris, and A. H. Jaffe. Robust forecasts on fundamental physics from the foregroundobscured, gravitationally-lensed CMB polarization. JCAP, 3:052, March 2016.

[41] G. D’Alessandro, A. Paiella, A. Coppolecchia, M.G. Castellano, I. Colantoni, P. de Bernardis, L. Lamagna, and S. Masi. Ultra high molecular weight polyethylene: Optical features at millimeter wavelengths. Infrared Physics \& Technology, 90:59 - 65, 2018. 
[42] D. Prêle, F. Voisin, M. Piat, T. Decourcelle, C. Perbost, C. Chapron, D. Rambaud, S. Maestre, W. Marty, and L. Montier. A 128 Multiplexing Factor Time-Domain SQUID Multiplexer. J. Low. Temp. Phys., 184(1-2):363-368, 2016.

[43] M. Salatino et al. Performance of NbSi transition-edge sensors readout with a 128 MUX factor for the QUBIC experiment. Proc. SPIE Int. Soc. Opt. Eng., 10708:1070845, 2018.

[44] P. K. Day, H. G. LeDuc, B. A. Mazin, A. Vayonakis, and J. Zmuidzinas. A broadband superconducting detector suitable for use in large arrays. Nature, 425:817-821, October 2003.

[45] F. Pajot et al. Planck Pre-Launch Status: HFI Ground Calibration. Astron. Astrophys., 520:A10, 2010.

[46] F. A. Bareiles, R. Morras, F. P. Hauscarriaga, J. C. Olalde, L. Guarrera, and E. M. Arnal. Alto Chorrillos: otra alternativa para instalar una antena para ondas milimétricas y submilimétricas en Argentina . BAAA, 53:199-202, September 2010.

[47] J. R. Pardo, J. Cernicharo, and E. Serabyn. Atmospheric transmission at microwaves (ATM): an improved model for millimeter/submillimeter applications. IEEE Transactions on Antennas and Propagation, 49:1683-1694, December 2001.

[48] A. Liu, M. Tegmark, S. Morrison, A. Lutomirski, and M. Zaldarriaga. Precision calibration of radio interferometers using redundant baselines. MNRAS, 408:1029-1050, October 2010.

[49] M.-A. Bigot-Sazy, R. Charlassier, J.-C. Hamilton, J. Kaplan, and G. Zahariade. Self-calibration: an efficient method to control systematic effects in bolometric interferometry. A\&A, 550:A59, February 2013.

[50] C. O'Sullivan, D. Burke, D. Gayer, J. D. Murphy, S. Scully et al. Simulations and performance of the QUBIC optical beam combiner. In Millimeter, Submillimeter, and Far-Infrared Detectors and Instrumentation for Astronomy IX, volume 10708 of Society of Photo-Optical Instrumentation Engineers (SPIE) Conference Series, page 107082I, July 2018.

\section{Bio}

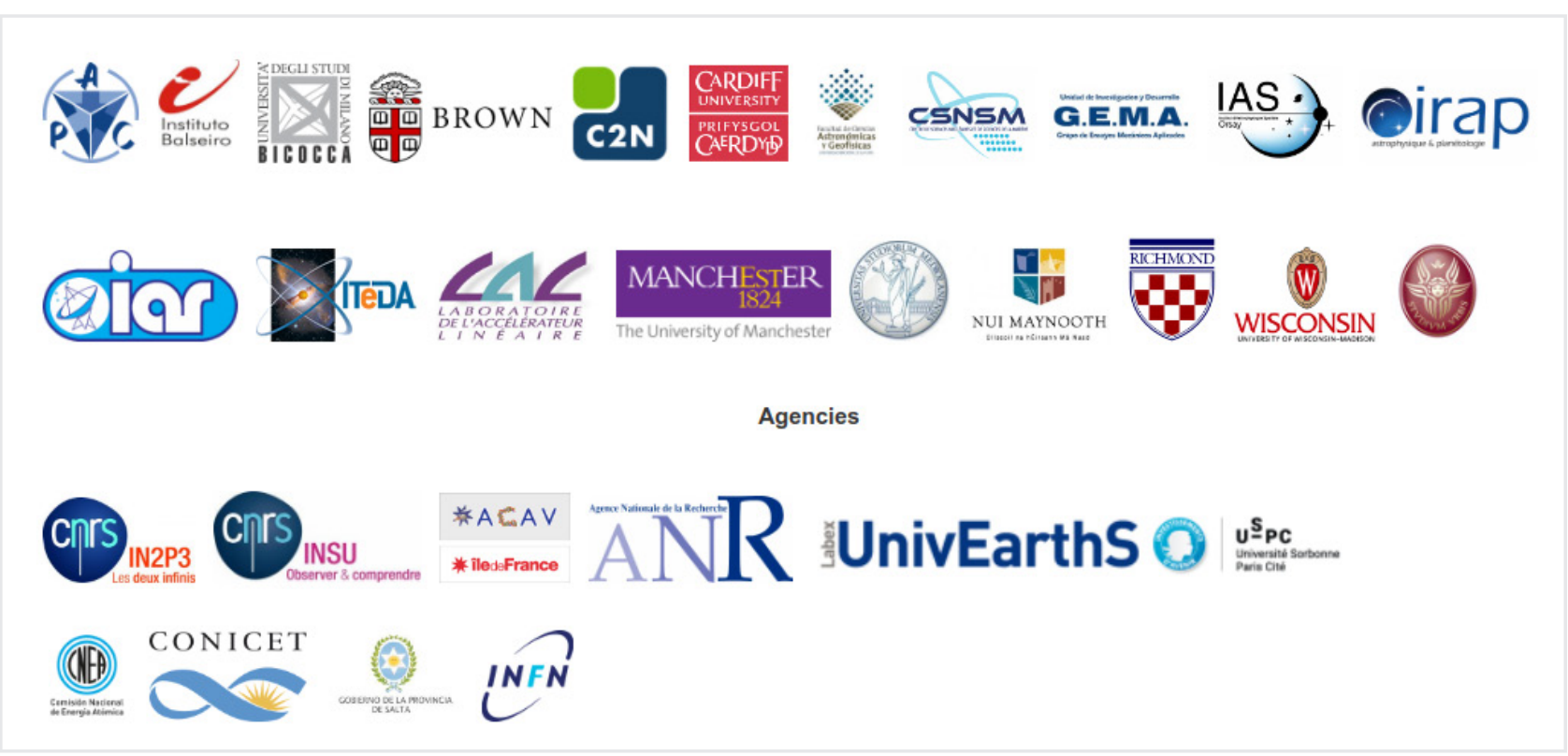

QUBIC is an international collaboration involving universities and laboratories in France, Italy, UK, Ireland, USA and Argentina. It is building an experiment aimed to reveal the existence of primordial gravitational waves through their effects upon the polarization of the cosmic microwave background, the relic radiation from the Big-Bang. The instrument will be installed in Alto Chorrillos, near San Antonio de los Cobres, Salta, Argentina. 